\title{
Motion in a Random Force Field
}

\author{
Dmitry Dolgopyat, Leonid Koralov \\ Department of Mathematics, University of Maryland \\ College Park, MD 20742 \\ dmitry@math.umd.edu, koralov@math.umd.edu
}

\begin{abstract}
We consider the motion of a particle in a random isotropic force field. Assuming that the force field arises from a Poisson field in $\mathbb{R}^{d}, d \geq 4$, and the initial velocity of the particle is sufficiently large, we describe the asymptotic behavior of the particle.
\end{abstract}

Mathematical Subject Classification: 60K37

\section{Introduction}

Let $F$ be a random force field on $\mathbb{R}^{d}$ defined on a probability space $\left(\Omega^{\prime}, \mathcal{F}^{\prime}, \mathrm{P}^{\prime}\right)$. The motion of a particle is described by the equation

$$
\ddot{X}(t)=F(X(t))
$$

where $X(t)$ denotes the position of the particle at time $t$. Let $V(t)=\dot{X}(t)$ be the velocity of the particle at time $t$. As initial conditions we take $X(0)=0$ and $V(0)=v_{0}$, where $v_{0}$ is a non-random vector. The force field is assumed to be stationary and isotropic. The precise form of the force field will be discussed below.

We shall be interested in the asymptotic behavior of $X(t)$ and $V(t)$ as $t \rightarrow \infty$. The process $V(t)$ can be written in the integral form as

$$
V(t)=v_{0}+\int_{0}^{t} F(X(s)) d s .
$$

Formal arguments, based on the near-independence of contributions to the integral on the right-hand side of (2) from non-intersecting sub-intervals, suggest that $V(t)$ behaves as a diffusion process, if time is re-scaled appropriately. In fact, we shall prove that there is an event $\Omega_{v_{0}}^{\prime}$ in the underlying probability space $\Omega^{\prime}$, such that $\mathrm{P}^{\prime}\left(\Omega^{\prime} \backslash \Omega_{v_{0}}^{\prime}\right) \rightarrow 0$ as $\left|v_{0}\right| \rightarrow \infty$, and $V\left(c^{3} t\right) / c$ converges, as $t \rightarrow \infty$, to a diffusion process on $\Omega_{v_{0}}^{\prime}$ (the probability measure 
on $\Omega_{v_{0}}^{\prime}$ is defined by conditioning $\mathrm{P}^{\prime}$ on the event $\Omega_{v_{0}}^{\prime}$ ). In particular, the kinetic energy of the particle will be shown to tend to infinity as $t \rightarrow \infty$. The precise formulation of these results will be provided in Section 3 ,

We cannot, however, expect that $V\left(c^{3} t\right) / c$ converges to the diffusion process for almost all realizations of the force field, if $v_{0}$ is fixed. Indeed, depending on the assumptions imposed on $F$, the trajectory may remain in a bounded region of space and the velocity may remain bounded with positive probability.

It must be noted that we must exclude the case $F=\nabla H$, where $H$ is a stationary field, since in this case $(X(t), V(t))$ is a Hamiltonian flow with the Hamiltonian $\bar{H}(k, x)=$ $|k|^{2} / 2-H(x)$, and $|V(t)|^{2} / 2-H(X(t))$ is constant on the solutions of (1).

Earlier papers primarily studied the behavior of $X(t)$ and $V(t)$ on long time intervals, whose length, however, depended on $\left|v_{0}\right|$, where the initial velocity $v_{0}$ was treated as a large parameter. We shall assume that $v_{0}$ is fixed and $t$ tends to infinity. The trade-off is that we need to exclude an event of small but positive measure from the underlying probability space.

Let us mention some of the earlier results concerning the long-time behavior of $X(t)$ and $V(t)$. In [5], Kesten and Papanicolaou considered the equation

$$
\ddot{x}(t)=\varepsilon F(x(t))
$$

with the initial data $x(0)=0$ and $v(0)=\widetilde{v}$. Certain mixing assumptions were imposed on the force field $F$. It was shown that if $d \geq 3$, the process $v\left(t / \varepsilon^{2}\right)$ converges weakly to a diffusion process $\bar{v}(t)$ with the initial data $\bar{v}(0)=\widetilde{v}$. The generator of the limiting process can be written out explicitly. The process $\varepsilon^{2} x\left(t / \varepsilon^{2}\right)$ converges weakly to $\bar{x}(t)=\int_{0}^{t} \bar{v}(s) d s$.

Note that equations (11) and (3) are related via the change of variables

$$
X(t)=x(t / \sqrt{\varepsilon}), \quad v_{0}=\widetilde{v} / \sqrt{\varepsilon} .
$$

Therefore, the convergence result for $v\left(t / \varepsilon^{2}\right)$ can be formulated in terms of $V(t)$ as follows: the process $V\left(\left|v_{0}\right|^{3} t\right) /\left|v_{0}\right|$ converges to a diffusion process when $v_{0}$ tends to infinity in such a way that $v_{0} /\left|v_{0}\right|=\widetilde{v}$ remains fixed. Similarly, $X\left(\left|v_{0}\right|^{3} t\right) /\left|v_{0}\right|^{4}$ converges weakly to a limiting process.

In [4, Durr, Goldstein, and Lebowitz extended the convergence results to the twodimensional case. The field $F$ was assumed to be a gradient of $H(x)=\sum_{i} h\left(x-p_{i}\right)$, where $h$ is a smooth function with compact support, and the points $p_{i}$ form a Poisson field on the plane. An additional difficulty in the two-dimensional case is that, unlike the case with $d \geq 3$, typical trajectories of (11) will self-intersect. In [7], Komorowski and Ryzhik proved the two-dimensional result in the case when $H$ is sufficiently mixing, but is not necessarily generated by a Poisson field.

In [6], Komorowski and Ryzhik considered the process (11) on a longer time scale. Namely, they demonstrated that $X\left(\left|v_{0}\right|^{3+8 \alpha} t\right) /\left|v_{0}\right|^{4(1+\alpha)}$ converges to a Brownian motion for all sufficiently small $\alpha>0$. It was assumed that $F=\nabla H$, where $H$ is sufficiently mixing. 
Unlike the above papers, we shall consider the asymptotic behavior of $V(t)$ when $v_{0}$ is fixed and $t \rightarrow \infty$. First, however, assume that $v_{0} \rightarrow \infty, v_{0} /\left|v_{0}\right|=\widetilde{v}$, and let $\bar{V}(t)$ be the limiting process for $V\left(\left|v_{0}\right|^{3} t\right) /\left|v_{0}\right|$ as $v_{0} \rightarrow \infty$. (It satisfies the stochastic differential equation (7), below.) As has been noted by Dolgopyat and De La Llave in [2], the process $\bar{V}(t)$ is self-similar, that is for $c>0$ the process $\bar{V}\left(c^{3} t\right) / c$ satisfies the same stochastic differential equation with initial condition $\bar{V}(0) / c$. Therefore, for $c$ fixed, $V\left(c^{3}\left|v_{0}\right|^{3} t\right) /\left(c\left|v_{0}\right|\right)$ tends to the diffusion process (7) starting at $\widetilde{v} / c$. If, instead, we assume that $v_{0}$ is large but fixed, and take the limit as $c \rightarrow \infty$, we formally obtain that $V\left(c^{3} t\right) / c$ tends to the diffusion process (77) starting at the origin. We remark that the diffusion processes satisfying the self-similarity property described above are well understood (see e.g. 9], Section XI). In particular the fact that this process is non-recurrent for $d>3$ plays a crucial role in our analysis.

\section{The Force Field}

Let $S_{R, m}$ be the space of smooth functions $f: \mathbb{R}^{d} \rightarrow \mathbb{R}^{d}$ which are supported inside the ball or radius $R$ centered at the origin and satisfy $\|f\|_{C^{2}\left(\mathbb{R}^{d}\right)} \leq m$. Let $\mu$ be a probability measure on $S_{R, m}$. We assume that $\mu$ is symmetric in the sense that if $\psi: S_{R, m} \rightarrow S_{R, m}$ is a mapping that maps a function $f$ into $-f$, then

$$
\mu(U)=\mu(\psi(U))
$$

for any measurable set $U \subseteq S_{R, m}$. We also assume that $\mu$ is isotropic, that is the vectors $\left(O^{-1} f\left(O x_{1}\right), \ldots, O^{-1} f\left(O x_{n}\right)\right)$ and $\left(f\left(x_{1}\right), \ldots, f\left(x_{n}\right)\right)$ have the same distribution for each orthogonal matrix $O$ and points $x_{1}, \ldots, x_{n} \in \mathbb{R}^{d}$. Suppose that on a probability space $\left(\Omega^{\prime}, \mathcal{F}^{\prime}, \mathrm{P}^{\prime}\right)$ we have a sequence of functions $f_{i}: \Omega^{\prime} \rightarrow S_{R, m}$ which are independent and identically distributed with distribution $\mu$. We shall consider random vector fields $F$ of the form

$$
F(x)=\sum_{i=1}^{\infty} f_{i}\left(x-r_{i}\right),
$$

where $r_{i}$ form a Poisson point field with unit intensity on $\mathbb{R}^{d}$. We assume that the Poisson field is independent of the sequence $f_{i}$. Note that the force field $F$ defined by ([6) is stationary, isotropic, and has zero mean. We shall denote the $j$-th coordinate of the vector $F$ by $F^{j}$. 


\section{Formulation of the Main Result}

Let $W_{t}$ be a standard $d$-dimensional Brownian motion. Consider the $d$-dimensional process $\bar{V}(t)$ which satisfies the diffusion equation

$$
d \bar{V}(t)=\frac{1}{\sqrt{|\bar{V}(t)|}}\left(\lambda d W_{t}+(\sigma-\lambda) \frac{\bar{V}(t)}{\left|\bar{V}^{2}(t)\right|}\left(\bar{V}(t), d W_{t}\right)\right)+\frac{\left((d-2) \sigma^{2}-(d-1) \lambda^{2}\right) \bar{V}(t)}{2|\bar{V}(t)|^{3}} d t
$$

where

$$
\sigma^{2}=\int_{-\infty}^{\infty} \mathrm{E}\left(F^{1}(0) F^{1}\left(e_{1} t\right)\right) d t, \quad \lambda^{2}=\int_{-\infty}^{\infty} \mathrm{E}\left(F^{2}(0) F^{2}\left(e_{1} t\right)\right) d t
$$

and $e_{1}$ is the first coordinate vector. It is clear that the integrals defining $\sigma^{2}$ and $\lambda^{2}$ are non-negative. We shall require that

$$
\int_{-\infty}^{\infty} \mathrm{E}\left(F^{1}(0) F^{1}\left(e_{1} t\right)\right) d t>0
$$

Thus, the case when $F=\nabla H$, where $H$ is a stationary random field, is excluded from consideration. The generator of the process $\bar{V}(t)$ is

$$
L=\frac{1}{2} \sum_{i, j=1}^{d} \frac{\partial}{\partial v_{i}} a_{i j}(v) \frac{\partial}{\partial v_{j}}
$$

where

$$
a_{i j}(v)=\int_{-\infty}^{\infty} \mathrm{E}\left(F^{i}(0) F^{j}(v t)\right) d t
$$

By examining the stochastic differential equation satisfied by $|\bar{V}(t)|^{2} / 2$ (see formula (10) below), it is it follows that the origin is an inaccessible point for the process $\bar{V}(t)$ if $d \geq 3$ (see [9], Section XI). Therefore the solution of (7) with initial condition $\bar{V}(0) \neq 0$ exists for all $t$. By the solution with the initial condition $\bar{V}(0)=0$ we shall mean the limit in distribution, as $\bar{V}(0) \rightarrow 0$, of solutions with initial condition $\bar{V}(0)$. We shall prove the following theorem.

Theorem 3.1. Let $F$ be a vector field in $\mathbb{R}^{d}, d \geq 4$, given by (6), which satisfies (9). For each sufficiently large $v_{0}$ there is a set $\Omega_{v_{0}}^{\prime}$ such that $\lim _{\left|v_{0}\right| \rightarrow \infty} \mathrm{P}^{\prime}\left(\Omega_{v_{0}}^{\prime}\right)=1$ and if $\Omega_{v_{0}}^{\prime}$ is viewed as a probability space with the measure obtained by conditioning $P^{\prime}$ on the event $\Omega_{v_{0}}^{\prime}$, i.e. $\mathrm{P}_{v_{0}}^{\prime}(A)=\mathrm{P}^{\prime}(A) / \mathrm{P}^{\prime}\left(\Omega_{v_{0}}^{\prime}\right)$, then

(a) the processes $X(t)$ and $V(t)$ tend to infinity almost surely,

(b) the processes $V\left(c^{3} t\right) / c$ on $\Omega_{v_{0}}^{\prime}$ converge in distribution, as $c \rightarrow \infty$, to the solution of (7) with the initial condition $\bar{V}(0)=0$. 
Let $E(t)=|V(t)|^{2} / 2$ be the kinetic energy of the particle at time $t$, and $\bar{E}(t)=$ $|\bar{V}(t)|^{2} / 2$, where $\bar{V}(t)$ is the solution of (7) with initial condition $\bar{V}(0)=0$. By the Ito formula, $\bar{E}(t)$ is the solution of

$$
d \bar{E}(t)=\sigma(2 \bar{E}(t))^{1 / 4} d B_{t}+\frac{\sigma^{2}(d-1)}{2 \sqrt{2 E}(t)} d t
$$

with the initial condition $\bar{E}(0)=0$, where $B_{t}$ is a standard one-dimensional Brownian motion. Let

$$
\bar{X}(t)=\int_{0}^{t} \bar{V}(s) d s .
$$

We observe that the fact that our force is Poisson and rotation invariant (rather than a general strongly mixing force) is primarily used in subsection 6.2. An alternative approach would be to estimate the rate of convergence in the averaging theorem (our Lemma 5.6) using the techniques of [3] or [6] but this would make the proof much more complicated. Therefore in this paper to we consider the simplest possible force distribution leaving the extension to more general force fields as an open question.

Remark. Up to a change of time by a constant factor, $\bar{E}^{3 / 4}(t)$, and consequently $|\bar{V}(t)|^{3 / 2}$, are Bessel processes with dimension 2d/3. Therefore if $d>3$, then from the properties of the Bessel processes (see Chapter 3.3.C of [8]) it follows that $\ln |\bar{V}(t)|$ is a diffusion process with a positive drift, and therefore

$$
\mathrm{P}\left(|\bar{V}(t)| \text { reaches } 2\left|v_{0}\right| \text { before }\left|v_{0}\right| / 2\right)>\frac{1}{2} \text {. }
$$

Moreover, $\quad \lim _{t \rightarrow \infty}|\bar{V}(t)|=\infty$ almost surely for $d>3$. We shall also see that $\lim _{t \rightarrow \infty}|V(t)|=\infty$ with high probability with respect to the measure $\mathrm{P}^{\prime}$ if the initial velocity is large (see Lemma 6.2). These properties will allow us to conclude that with high probability the trajectories of $\bar{X}(t)$ and $X(t)$ do not "come close" to self-intersecting if the initial velocity is large (see Lemma 6.5). This avoidance of near self-intersections is essential to the proof of Theorem 3.1.

Theorem 3.1 immediately implies the following.

Corollary 3.2. Let $F$ be a vector field in $\mathbb{R}^{d}, d \geq 4$, given by (6), which satisfies (9). For each sufficiently large $v_{0}$ there is a set $\Omega_{v_{0}}^{\prime}$ such that $\lim _{\left|v_{0}\right| \rightarrow \infty} \mathrm{P}^{\prime}\left(\Omega_{v_{0}}^{\prime}\right)=1$ and if $\Omega_{v_{0}}^{\prime}$ is viewed as a probability space with the measure obtained by conditioning $P^{\prime}$ on the event $\Omega_{v_{0}}^{\prime}$, then

(a) the processes $E\left(c^{3} t\right) / c^{2}$ on $\Omega_{v_{0}}^{\prime}$ converge in distribution, as $c \rightarrow \infty$, to the solution of (10) with the initial condition $\bar{E}(0)=0$. The processes $X\left(c^{3} t\right) / c^{4}$ on $\Omega_{v_{0}}^{\prime}$ converge in distribution, as $c \rightarrow \infty$, to the process $\bar{X}(t)$ defined by (11).

(b) There exists a constant $\bar{c}$ such that $E(t) / \bar{c} t^{2 / 3}$ converges in distribution to a random variable with density

$$
p(x)=\frac{3}{2 \Gamma(d / 3)} x^{\frac{d}{2}-1} \exp \left(-x^{\frac{3}{2}}\right) .
$$


If $d=2$ or 3 then the situation is more delicate since $\bar{V}$ is recurrent. it seems that the methods of the present paper can be modified to show that $P(|\dot{Y}(t)| \rightarrow \infty)=0$ (in the more difficult case $d=2$ where the trajectories of $X(t)$ self sintersect the methods of [4, 7] should be used). We also beleive that Theorem 3.1(b) remains valid for $d=2,3$ (the theorem is false if $d=1$ since in that case all orbits are periodic). However we do not have a proof of this since the cases of large $V$ and small $V$ need to be considered separately and new ideas are necessary to handle the latter case.

\section{Auxiliary Processes}

\subsection{Time Discretization}

Let $X(t)$ be the solution of (1) with initial conditions $X(0)=0, V(0)=v_{0}$. We assume that the field $F$ and, consequently, the process $X(t)$ are defined on a probability space $\left(\Omega^{\prime}, \mathcal{F}^{\prime}, \mathrm{P}^{\prime}\right)$. Assume, momentarily, that the trajectories of $X(t)$ always "keep exploring" new regions of $\mathbb{R}^{d}$ in the sense that for each $t \geq 0$ the tail of the trajectory $X(s), s \geq t+1$, is separated from the initial part of the trajectory $X(s), s \leq t$, by a distance larger than $2 R$. Then, for large $t$, the interval $[0, t]$ can be split into sub-intervals, such that the contribution to the integral on the right-hand side of (2) from different sub-intervals are almost independent. This fact will be helpful when proving that $V\left(c^{3} t\right) / c$ converges to a diffusion process.

We shall demonstrate that with high probability the trajectories of the process $X(t)$ indeed have the desired property if the initial velocity is large. To this end, we shall construct an auxiliary process $Y(t)$ on a probability space $(\Omega, \mathcal{F}, \mathrm{P})$. The process $Y(t)$ is defined as the solution of

$$
\ddot{Y}(t)=\widetilde{F}(t, Y(t)), \quad Y(0)=0, \dot{Y}(0)=v_{0},
$$

where $\widetilde{F}(t, x)$ can be obtained from $F(x)$ by "switching on" new independent versions of $F(x)$ at stopping times $\tau_{n}$, as described below. Since the force field $\widetilde{F}$ on the right-hand side of (13) is time-dependent, the increments $\dot{Y}\left(\tau_{n}\right)-\dot{Y}\left(\tau_{n-1}\right)$ and $\dot{Y}\left(\tau_{k}\right)-\dot{Y}\left(\tau_{k-1}\right)$ will be almost independent if $|n-k|$ is large. This way, we don't need to be concerned about possible self-intersections of the process $Y(t)$ when studying the long-time behavior of the process $\dot{Y}(t)$. Moreover, the introduction of the stopping times $\tau_{n}$ will allow us to use a kind of Markov property: the distribution of $Y\left(\tau_{n}+\cdot\right)-Y\left(\tau_{n}\right)$ will depend on the events prior to $\tau_{n}$ only through $\dot{Y}\left(\tau_{n}\right)$ (see Section 4.2 below).

On the other hand, we shall prove that the processes $X(t)$ and $Y(t)$ will have the same distribution if certain events with small probabilities are excluded from their respective probability spaces. More precisely, there are events $\Omega_{v_{0}}^{\prime} \subseteq \Omega^{\prime}$ and $\Omega_{v_{0}} \subseteq \Omega$ such that the processes $X\left(t, \omega^{\prime}\right) \chi_{\Omega_{v_{0}}}\left(\omega^{\prime}\right)$ and $Y(t, \omega) \chi_{\Omega_{v_{0}}}(\omega)$ have the same distributions. The probabilities of $\Omega_{v_{0}}^{\prime}$ and $\Omega_{v_{0}}$ tend to one when $\left|v_{0}\right| \rightarrow \infty$. 
Below we give a rigorous definition of the field $\widetilde{F}(t, x)$. Roughly speaking, we follow the trajectory $X(t)$ till time $\tau_{1}$ such that there are no points $r_{i}, i \geq 1$, in the $2 R$-neighborhood of $X\left(\tau_{1}\right)$. Then we replace the force field $F$ by an independent version, also generated by Poisson points with unit intensity on $\mathbb{R}^{d} \backslash B_{2 R}\left(X\left(\tau_{1}\right)\right)$, but zero intensity on $B_{2 R}\left(X\left(\tau_{1}\right)\right)$, where $B_{2 R}\left(X\left(\tau_{1}\right)\right)$ is the ball of radius $2 R$ centered at the $X\left(\tau_{1}\right)$. We can then treat $X\left(\tau_{1}\right)$ as the new initial point and define the following stopping times by induction. More precisely, let $i, n \geq 1$, and $f_{i}^{n}$ be independent identically distributed functions with distribution $\mu$. Let $F_{0}=F$. Define the sequence of random fields $F_{1}, F_{2}, \ldots$ as follows:

$$
F_{n}(x)=\sum_{i=1}^{\infty} f_{i}^{n}\left(x-r_{i}^{n}\right),
$$

where, for each $n \geq 1, r_{i}^{n}$ form a Poisson point field with unit intensity on $\mathbb{R}^{d} \backslash B_{2 R}(0)$ and zero intensity on $B_{2 R}(0)$, and $B_{2 R}(0)$ is the ball of radius $2 R$ centered at the origin. The Poisson fields $r^{0}, r^{1}, r^{2}, \ldots$ are assumed to be independent of each other and of $f_{i}^{n}$ (here $\left.r^{0}=r\right)$. We can assume that the random fields $F_{n}$ are defined on a probability space $(\Omega, \mathcal{F}, \mathrm{P})$, which is an extension of the original probability space $\left(\Omega^{\prime}, \mathcal{F}^{\prime}, \mathrm{P}^{\prime}\right)$. Let $\tau_{0}=0$, $\widetilde{F}_{0}=F_{0}, Y_{0}=0$, and $v_{0}$ be the initial condition for the process $X(t)$. Assuming that $\tau_{n-1}$, $\widetilde{F}_{n-1}, Y_{n-1}$, and $v_{n-1}$ have been defined for some $n \geq 1$, we inductively define $\tau_{n}, \widetilde{F}_{n}, Y_{n}$, and $v_{n}$. Let $y(t)$ be the solution of the equation

$$
\ddot{y}(t)=\widetilde{F}_{n-1}(y(t)), \quad t \geq \tau_{n-1}
$$

with the initial conditions $y\left(\tau_{n-1}\right)=Y_{n-1}, \dot{y}\left(\tau_{n-1}\right)=v_{n-1}$. Let $l=4 R+1$. Let $\tau_{n}$ be the first time after $\tau_{n-1}+l\left|v_{n-1}\right|^{-1}$ when there are no points $r_{i}^{n-1}, i \geq 1$, within the $2 R$-neighborhood of $y(t)-Y_{n-1}$, that is

$$
\tau_{n}=\inf \left\{t \geq \tau_{n-1}+l\left|v_{n-1}\right|^{-1}: \inf _{i \geq 1}\left|y(t)-Y_{n-1}-r_{i}^{n-1}\right| \geq 2 R\right\}
$$

If $\tau_{n}=\infty$, then $Y_{i}, v_{i}$ and $\widetilde{F}_{i}(x)$ are undefined for $i \geq n$. Otherwise, define $Y_{n}=y\left(\tau_{n}\right)$, $v_{n}=\dot{y}\left(\tau_{n}\right)$, and $\widetilde{F}_{n}(x)=F_{n}\left(x-Y_{n}\right)$.

Now we can set $\widetilde{F}(t, x)=\widetilde{F}_{n-1}(x)$ for $\tau_{n-1} \leq t<\tau_{n}$. Then the solution $Y(t)$ of (13) satisfies $Y\left(\tau_{n}\right)=Y_{n}$ and $Y\left(\tau_{n}\right)=v_{n}$. The relation of $Y(t)$ to the original process $X(t)$ is explained by the following lemma.

Lemma 4.1. Let $Y(t)$ be the solution of (13) on the probability space $(\Omega, \mathcal{F}, \mathrm{P})$. For each sufficiently large $v_{0}$ there are events $\Omega_{v_{0}}^{\prime} \subseteq \Omega^{\prime}$ and $\Omega_{v_{0}} \subseteq \Omega$ with the following properties:

(a) $\lim _{\left|v_{0}\right| \rightarrow \infty} \mathrm{P}^{\prime}\left(\Omega_{v_{0}}^{\prime}\right)=\lim _{\left|v_{0}\right| \rightarrow \infty} \mathrm{P}\left(\Omega_{v_{0}}\right)=1$.

(b) The processes $X(t)$ and $Y(t)$ have the same distribution if restricted to the spaces $\Omega_{v_{0}}^{\prime}$ and $\Omega_{v_{0}}$, respectively.

(c) The processes $\dot{Y}(t)$ and $Y(t)$ tend to infinity almost surely on $\Omega_{v_{0}}$.

(d) If $\Omega_{v_{0}}$ is viewed as a probability space with the measure obtained by conditioning $P$ on the event $\Omega_{v_{0}}$, then the processes $\dot{Y}\left(c^{3} t\right) / c$ on $\Omega_{v_{0}}$ converge in distribution, as $c \rightarrow \infty$, to the solution of (7) with the initial condition $\bar{V}(0)=0$. 
It is clear that Theorem 3.1 follows from Lemma 4.1. We shall study some of the properties of $Y(t)$ in Section 5 and prove parts (a), (b) and (c) of Lemma 4.1 in Section 6 , We then prove part (d) of Lemma 4.1 in Section 7.

\subsection{Another Auxiliary Process}

Note that the distribution of vector field $F_{0}$ is slightly different from the distribution of the fields $F_{n}, n \geq 1$. Namely, $F_{0}$ is a based on a Poisson field on $\mathbb{R}^{d}$, while $F_{n}, n \geq 1$, are based on Poisson fields on $\mathbb{R}^{d} \backslash B_{2 R}(0)$.

Consider the vector field $\bar{F}$, which is defined in the same way as $\widetilde{F}$, except that now we assume $F_{0}$ to be defined by a Poisson field with unit intensity on $\mathbb{R}^{d} \backslash B_{2 R}(0)$ and zero intensity on $B_{2 R}(0)$. The process $Z(t)$ is defined as the solution of

$$
\ddot{Z}(t)=\bar{F}(t, Z(t)), \quad Z(0)=0, \dot{Z}(0)=w_{0},
$$

where $w_{0}$ is a random vector independent of $\bar{F}$. The reason to consider $Z(t)$ is the following Markov property.

Let $\mathcal{G}_{n}$ be the $\sigma$-algebra generated by $\widetilde{F}_{i}, i \leq n-1$. For each $n \geq 1, A \in \mathcal{B}\left(\mathbb{R}^{d}\right)$, and $B \in \mathcal{B}(C([0, \infty)))$ we have:

$$
\mathrm{P}\left(Y\left(\tau_{n}+\cdot\right)-Y\left(\tau_{n}\right) \in B \mid \mathcal{G}_{n}\right) \chi_{\left\{\dot{Y}\left(\tau_{n}\right) \in A\right\}}=\mathrm{P}(Z(\cdot) \in B) \chi_{\left\{w_{0} \in A\right\}} \text { in distribution, }
$$

where the initial velocity vector $w_{0}$ for the process $Z(t)$ is assumed to be distributed as $\dot{Y}\left(\tau_{n}\right)$.

If a random variable $\tau$ is such that $\tau(\omega) \in\left\{\tau_{1}(\omega), \tau_{2}(\omega), \ldots\right\}$ for each $\omega$, and the set $\left\{\tau \leq \tau_{n}\right\}$ is $\mathcal{G}_{n}$-measurable for each $n$, then from (14) it follows that

$$
\mathrm{P}(Y(\tau+\cdot)-Y(\tau) \in B \mid \mathcal{G}) \chi_{\{\dot{Y}(\tau) \in A\}}=\mathrm{P}(Z(\cdot) \in B) \chi_{\left\{w_{0} \in A\right\}} \text { in distribution, }
$$

where $\mathcal{G}=\left\{A \in \mathcal{F}: A \cap\left\{\tau \leq \tau_{n}\right\} \in \mathcal{G}_{n}\right.$ for each $\left.n\right\}$.

\section{Preliminaries}

In this section we recall some results about diffusion approximation for the process $\dot{Y}(t)$ and provide bounds on probabilities of some unlikely events.

\subsection{Behavior of $Y(t)$ and $\dot{Y}(t)$ on the Time Interval $\left[\tau_{n}, \tau_{n+1}\right]$}

In this subsection we shall prove that with high probability the velocity vector does not change significantly between the times $\tau_{n}$ and $\tau_{n+1}$ if $\left|v_{n}\right|$ is large. Therefore $Y(t)$ can be well approximated by a straight line on this time interval.

Let $z_{n}(t)=Y_{n}+\left(t-\tau_{n}\right) v_{n}$, that is $z_{n}(t)$ is the solution of

$$
\ddot{z}_{n}(t)=0, \quad z_{n}\left(\tau_{n}\right)=Y_{n}, \dot{z}_{n}\left(\tau_{n}\right)=v_{n} .
$$


Let $\eta_{n}, n \geq 1$ be the first time after $\tau_{n-1}+l\left|v_{n-1}\right|^{-1}$ when there are no points $r_{i}^{n-1}, i \geq 1$, within the $2 R$-neighborhood of $z_{n-1}(t)-Y_{n-1}$, that is

$$
\eta_{n}=\inf \left\{t \geq \tau_{n-1}+l\left|v_{n-1}\right|^{-1}: \inf _{i \geq 1}\left|z_{n-1}(t)-Y_{n-1}-r_{i}^{n-1}\right| \geq 2 R\right\}
$$

Let

$$
\xi_{n}(t)=\int_{\tau_{n}}^{t} \widetilde{F}_{n}\left(z_{n}(s)\right) d s, \quad \zeta_{n}(t)=\int_{\tau_{n}}^{t} \xi_{n}(s) d s, \quad t \geq \tau_{n} .
$$

Let us first examine the behavior of $Y(t)$ on the interval $\left[0, \tau_{1}\right]$.

Lemma 5.1. For each $N$ and $\delta>0$ we have

$$
\begin{gathered}
\mathrm{P}\left(\tau_{1}>\left|v_{0}\right|^{-1+\delta}\right) \leq\left|v_{0}\right|^{-N} \\
\mathrm{P}\left(\sup _{0 \leq t \leq \tau_{1}}\left|\dot{Y}(t)-v_{0}\right|>\left|v_{0}\right|^{-1+\delta}\right) \leq\left|v_{0}\right|^{-N}, \\
\mathrm{P}\left(\sup _{0 \leq t \leq \tau_{1}}\left|\dot{Y}(t)-v_{0}-\xi_{0}(t)\right|>\left|v_{0}\right|^{-3+\delta}\right) \leq\left|v_{0}\right|^{-N}, \\
\mathrm{P}\left(\left|\xi_{0}\left(\tau_{1}\right)-\int_{0}^{\eta_{1}} F_{0}\left(z_{0}(s)\right) d s\right|>\left|v_{0}\right|^{-1+\delta}\right) \leq\left|v_{0}\right|^{-N},
\end{gathered}
$$

for all sufficiently large $\left|v_{0}\right|$.

Proof. Let $y(t), t \geq 0$, be the solution of the equation

$$
\ddot{y}(t)=F_{0}(y(t)), \quad y(0)=0, \dot{y}(0)=v_{0},
$$

Note that $Y(t)$ satisfies this equation on the interval $\left[0, \tau_{1}\right)$.

We shall say that an event (which depends on $v_{0}$ ) happens with high probability if for each $N$ the probability of the complement does not exceed $\left|v_{0}\right|^{-N}$ for all sufficiently large $\left|v_{0}\right|$. Let us show that for each $\delta>0$

$$
\left\|F_{0}\right\|_{C^{2}\left(B_{\left|v_{0}\right|}(0)\right)} \leq \delta \ln \left|v_{0}\right|
$$

with high probability. Recall the definition of $F_{0}=F$ from Section 2 , and note that $\left\|F_{0}\right\|_{C^{2}\left(B_{\left|v_{0}\right|}(0)\right)}$ may be larger than $\delta \ln \left|v_{0}\right|$ only if there is a point $x \in B_{\left|v_{0}\right|}(0)$ such that the ball of radius $R$ centered at $x$ contains at least $\left[\delta \ln \left|v_{0}\right|\right] / m$ points out of $r_{i}, i \geq 1$. The probability of this event is easily seen to decay faster than any power of $\left|v_{0}\right|$ since $\delta$, $m$ and $R$ are constants and $r_{i}, i \geq 1$, form a Poisson field with unit intensity.

Take $-1<\alpha<0$, which will be specified later, and let $T_{0}=\left|v_{0}\right|^{\alpha}$. Let us show that for each $\delta>0$

$$
\sup _{0 \leq t \leq T_{0}}\left|\xi_{0}(t)\right| \leq\left|v_{0}\right|^{(\alpha-1) / 2+\delta}
$$


with high probability. Indeed, let

$$
\bar{\xi}^{k}=\xi_{0}\left(\frac{3(k+1) R}{\left|v_{0}\right|}\right)-\xi_{0}\left(\frac{3 k R}{\left|v_{0}\right|}\right), \quad k \geq 0
$$

where $R$ was defined in Section 2. Note that $\left\{\bar{\xi}^{2 k}\right\}_{k \geq 0}$ and $\left\{\bar{\xi}^{2 k+1}\right\}_{k \geq 0}$ are sequences of independent identically distributed random variables since the force field is uncorrelated at distances larger than $2 R$. Their tails decay faster than exponentially since $F$ is based on a Poisson random field. Therefore, the moderate deviation bounds (see, for example, Theorem 9.4 of [1]) imply that

$$
\sup _{m \leq\left|v_{0}\right|^{\alpha+1} / 3 R}\left(\left|\sum_{k=0}^{m} \bar{\xi}^{2 k}\right|+\left|\sum_{k=0}^{m} \bar{\xi}^{2 k+1}\right|\right) \leq\left|v_{0}\right|^{-1}\left|v_{0}\right|^{\frac{\alpha+1}{2}+\delta}
$$

since the standard deviation of $\bar{\xi}^{k}$ is of order $1 /\left|v_{0}\right|$. This easily implies (22) since $T_{0}=\left|v_{0}\right|^{\alpha}$.

From (22) it immediately follows that for each $\delta>0$

$$
\sup _{0 \leq t \leq T_{0}}\left|\zeta_{0}(t)\right| \leq\left|v_{0}\right|^{(3 \alpha-1) / 2+\delta}
$$

with high probability. Let $\sigma=\inf \left\{t: y(t) \notin B_{\left|v_{0}\right|}(0)\right\}$ (with the convention that the infimum of the empty set is $+\infty)$. By (201),

$$
y(t)=z_{0}(t)+\zeta_{0}(t)+\int_{0}^{t} \int_{0}^{u}\left(F_{0}(y(s))-F_{0}\left(z_{0}(s)\right)\right) d s d u .
$$

Therefore,

$$
\left|y(t)-z_{0}(t)\right| \leq\left|\zeta_{0}(t)\right|+T_{0}|| F_{0} \|_{C^{1}\left(B_{\left|v_{0}\right|}(0)\right)} \int_{0}^{t}\left|y(s)-z_{0}(s)\right| d s \quad \text { for } \quad 0 \leq t \leq T_{0} \wedge \sigma
$$

By (21) and (23), this implies that for each $\delta>0$,

$$
\sup _{0 \leq t \leq T_{0} \wedge \sigma}\left|y(t)-z_{0}(t)\right| \leq\left|v_{0}\right|^{(3 \alpha-1) / 2+\delta}+\delta\left|v_{0}\right|^{2 \alpha} \ln \left|v_{0}\right| \sup _{0 \leq t \leq T_{0} \wedge \sigma}\left|y(t)-z_{0}(t)\right|
$$

with high probability. Since $\delta\left|v_{0}\right|^{2 \alpha} \ln \left|v_{0}\right|<1 / 2$ for large enough $\left|v_{0}\right|$, this implies that

$$
\sup _{0 \leq t \leq T_{0} \wedge \sigma}\left|y(t)-z_{0}(t)\right| \leq 2\left|v_{0}\right|^{(3 \alpha-1) / 2+\delta}
$$

with high probability and, consequently, $\sigma>T_{0}$ with high probability.

Since $\delta$ was an arbitrary positive number, we obtain that for each $\delta>0$

$$
\sup _{0 \leq t \leq T_{0}}\left|y(t)-z_{0}(t)\right| \leq\left|v_{0}\right|^{(3 \alpha-1) / 2+\delta}
$$


with high probability. By (20),

$$
\dot{y}(t)-v_{0}=\xi_{0}(t)+\int_{0}^{t}\left(F_{0}(y(s))-F_{0}\left(z_{0}(s)\right)\right) d s .
$$

Due to (22) and (24), for each $\delta>0$

$$
\sup _{0 \leq t \leq T_{0}}\left|\dot{y}(t)-v_{0}\right| \leq\left|v_{0}\right|^{(\alpha-1) / 2+\delta}
$$

with high probability. By the expression $\left\langle\nabla F_{0}, v\right\rangle$, where $v$ is a vector, we shall mean the vector $w$ with components $w^{j}=\sum_{i=1}^{d} F_{0 x_{i}}^{j} v^{i}$. If $y(t) \in B_{\left|v_{0}\right|}(0)$ for $0 \leq t \leq T_{0}$, then by the Taylor formula

$$
\begin{gathered}
\sup _{0 \leq t \leq T_{0}}\left|\dot{y}(t)-v_{0}-\xi_{0}(t)\right| \leq \\
\leq \sup _{0 \leq t \leq T_{0}}\left|\int_{0}^{t}\left\langle\nabla F_{0}\left(z_{0}(s)\right),\left(y(s)-z_{0}(s)\right)\right\rangle d s\right|+\sup _{0 \leq t \leq T_{0}} \frac{1}{2} \int_{0}^{t}\left\|F_{0}\right\|_{C^{2}\left(B_{\left|v_{0}\right|}(0)\right)}\left|y(s)-z_{0}(s)\right|^{2} d s .
\end{gathered}
$$

From (21) and (24) it follows that for each $\delta>0$ the second term in the right-hand side does not exceed $\left|v_{0}\right|^{4 \alpha-1+\delta}$ with high probability. To estimate the first term we use the fact that

$$
\sup _{0 \leq t \leq T_{0}}\left|\int_{0}^{t} F_{0 x_{i}}^{j}\left(z_{0}(s)\right) d s\right| \leq\left|v_{0}\right|^{(\alpha-1) / 2+\delta}, \quad 1 \leq i, j \leq d,
$$

with high probability. Then, after integrating by parts and using (24) and (25), we obtain that the first term in the right-hand side does not exceed $\left|v_{0}\right|^{2 \alpha-1+\delta}$ with high probability. Therefore, for each $\delta>0$

$$
\sup _{0 \leq t \leq T_{0}}\left|\dot{y}(t)-v_{0}-\xi_{0}(t)\right| \leq\left|v_{0}\right|^{2 \alpha-1+\delta}
$$

with high probability.

Note that the set $\left\{z_{0}(t), t \in\left[T_{0} / 4, T_{0} / 2\right]\right\}$ is a straight segment of length $\left|v_{0}\right|^{1+\alpha} / 4$, and the points $r_{i}^{0}, i \geq 1$, form a Poisson field. This implies that with high probability there is a moment of time $t \in\left[T_{0} / 4, T_{0} / 2\right]$ such that there are no points $r_{i}^{0}, i \geq 1$, within the $4 R$-neighborhood of $z_{0}(t)$. Therefore,

$$
\eta_{1} \leq T_{0}
$$

with high probability. Moreover, from the proximity of $y(t)$ and $z_{0}(t)$ (formula (24)) and the definition of $\tau_{1}$ it now follows

$$
\tau_{1} \leq T_{0}
$$

with high probability. Since $\alpha \in(0,1)$ was arbitrary, this implies (16) $)$. Combining (28) with (25) and (26), we obtain (17) and (18), respectively. Combining (27) and (28) with (22), we obtain that for arbitrary $\delta>0$ we have

$$
\left|\int_{0}^{\eta_{1}} F_{0}\left(z_{0}(s)\right) d s\right|+\left|\xi_{0}\left(\tau_{1}\right)\right| \leq\left|v_{0}\right|^{-1+\delta}
$$


with high probability, which implies (19).

Remark. Obviously, the same result holds if the process $Y(t)$ is replaced by the process $Z(t)$ with initial velocity $v_{0}$. Therefore, we have the following.

Corollary 5.2. For each $N$ and $\delta>0$ there is $r>0$ such that for each $n$

$$
\begin{gathered}
\mathrm{P}\left(\tau_{n+1}-\tau_{n}>\left|v_{n}\right|^{-1+\delta} \mid \mathcal{G}_{n}\right) \leq\left|v_{n}\right|^{-N}, \\
\mathrm{P}\left(\sup _{\tau_{n} \leq t \leq \tau_{n+1}}\left|\dot{Y}(t)-v_{n}\right|>\left|v_{n}\right|^{-1+\delta} \mid \mathcal{G}_{n}\right) \leq\left|v_{n}\right|^{-N}, \\
\mathrm{P}\left(\sup _{\tau_{n} \leq t \leq \tau_{n+1}}\left|\dot{Y}(t)-v_{n}-\xi_{n}(t)\right|>\left|v_{n}\right|^{-3+\delta} \mid \mathcal{G}_{n}\right) \leq\left|v_{n}\right|^{-N}, \\
\mathrm{P}\left(\left|\xi_{n}\left(\tau_{n+1}\right)-\int_{\tau_{n}}^{\eta_{n+1}} \widetilde{F}_{n}\left(z_{n}(s)\right) d s\right|>\left|v_{n}\right|^{-1+\delta} \mid \mathcal{G}_{n}\right) \leq\left|v_{n}\right|^{-N},
\end{gathered}
$$

hold almost surely on the event $\left|v_{n}\right|>r$.

Let $H_{n}$ be the following event

$$
H_{n}=\left\{\left|\dot{Y}\left(\tau_{n+1}\right)-\dot{Y}\left(\tau_{n}\right)-\int_{\tau_{n}}^{\eta_{n+1}} \widetilde{F}_{n}\left(z_{n}(s)\right) d s\right| \leq\left|v_{n}\right|^{-1+\delta}\right\} .
$$

The following Lemma will be proved in the Appendix.

Lemma 5.3. For each $\delta>0$ we have

$$
\mathrm{E}\left(\chi_{H_{0}}\left|\dot{Y}\left(\tau_{1}\right)-v_{0}-\int_{0}^{\eta_{1}} F_{0}\left(z_{0}(s)\right) d s\right|\right) \leq\left|v_{0}\right|^{-3+\delta}
$$

for all sufficiently large $\left|v_{0}\right|$.

For each $\delta>0$ there is $r>0$ such that for each $n$

$$
\mathrm{E}\left(\chi_{H_{n}}\left|\dot{Y}\left(\tau_{n+1}\right)-\dot{Y}\left(\tau_{n}\right)-\int_{\tau_{n}}^{\eta_{n+1}} \widetilde{F}_{n}\left(z_{n}(s) d s\right)\right| \mid \mathcal{G}_{n}\right) \leq\left|v_{n}\right|^{-3+\delta}
$$

almost surely on the event $\left|v_{n}\right|>r$.

\subsection{Behavior of $Y(t)$ and $\dot{Y}(t)$ on a Time Interval Proportional to $\left|v_{0}\right|^{3}$}

Recall that $x(t)=X\left(t /\left|v_{0}\right|\right)$ satisfies (3) with $\varepsilon=1 /\left|v_{0}\right|^{2}$ and initial data $x(0)=0, \dot{x}(0)=$ $v_{0} /\left|v_{0}\right|$. As discussed in the Introduction, the scale on which we see the diffusion for $\dot{x}(t)$ is of order $t \sim 1 / \varepsilon^{2}=\left|v_{0}\right|^{4}$. Since $\dot{x}\left(\left|v_{0}\right| t\right)=\dot{X}(t) /\left|v_{0}\right|$, one needs time of order $t \sim\left|v_{0}\right|^{3}$ to see fluctuations of order one for the process $\dot{X}(t) /\left|v_{0}\right|$. 
In this section we recall the effective equation for $\dot{Y}$ on the scale $\left|v_{0}\right|^{3}$ and provide estimates for the probability that $\dot{Y}$ changes much faster or much slower than expected.

For $a, r>0, b>1$, and $n \geq 0$, let

$$
\begin{gathered}
\hat{\tau}_{n}^{a}=\min _{k \geq n}\left\{\tau_{k}: \tau_{k}-\tau_{n} \geq a r^{3}\right\}, \\
\check{\tau}_{n}^{b}=\min _{k \geq n}\left\{\tau_{k}:|\dot{Y}(t)| \notin(r, b r) \text { for some } \tau_{n} \leq t \leq \tau_{k}\right\}, \\
\bar{\tau}_{n}=\min \left\{\hat{\tau}_{n}^{a}, \check{\tau}_{n}^{b}\right\} .
\end{gathered}
$$

(As always, the minimum over the empty set is $+\infty$.) In what follows $a$ and $b$ will be fixed. The constant $r$ will serve as a large parameter, and $\left|v_{0}\right|$ will be assumed to be of order $r$. Thus $\hat{\tau}_{0}^{a}$ is the first of the stopping times $\tau_{k}$ which is larger than $a r^{3}$. Roughly speaking, $\hat{\tau}_{0}^{a}$ is very close to $a r^{3}$. The stopping time $\check{\tau}_{0}^{b}$ is, roughly speaking, the first time when $|\dot{Y}(t)|$ changes from $\left|v_{0}\right|$ to either $r$ or $b r$ (assuming that $\left|v_{0}\right| \in(r, b r)$ ).

Assume that $r$ is large and $\left|v_{0}\right| \in(r, b r)$. Let us first describe the behavior of the process $Y(t)$ on the time interval $\left[0, \bar{\tau}_{0}\right]$.

Lemma 5.4. For each $N, \delta>0, a>0$, and $b>1$, we have

$$
\begin{gathered}
\mathrm{P}\left(\tau_{n+1}-\tau_{n}>\left|v_{n}\right|^{-1+\delta} \text { for some } n \text { such that } \tau_{n}<\bar{\tau}_{0}\right) \leq r^{-N} \\
\mathrm{P}\left(\sup _{\tau_{n} \leq t \leq \tau_{n+1}}\left|\dot{Y}(t)-v_{n}\right|>\left|v_{n}\right|^{-1+\delta} \text { for some } n \text { such that } \tau_{n}<\bar{\tau}_{0}\right) \leq r^{-N} \\
\mathrm{P}\left(\bar{\tau}_{0}=\infty\right) \leq r^{-N}
\end{gathered}
$$

for all sufficiently large $r$ (i.e. for all $r \geq r_{0}$, where $r_{0}$ depends on the distribution of the force field and on $N, \delta, a$ and $b$ ) and all $\left|v_{0}\right| \in(r, b r)$.

Proof. For fixed $n$, the probability $\mathrm{P}\left(\tau_{n+1}-\tau_{n}>\left|v_{n}\right|^{-1+\delta}, \tau_{n}<\bar{\tau}_{0}\right)$ is estimated from above by $r^{-N}$ due to (16) (if $n=0$ ) and (29) (if $n \geq 1$ ). The number of $n$ for which $\tau_{n}<\bar{\tau}_{0}$ does not exceed $a b r^{4}$. Since $N$ was arbitrary, this implies (35). In the same way, (17) and (30) imply (36). Finally, (35) implies (37) again due to the fact that $\tau_{n} \geq \bar{\tau}_{0}$ for $n>a b r^{4}$.

As before, by considering $Z(t)$ instead of $Y(t)$, we obtain the following.

Corollary 5.5. For each $N, \delta>0, a>0$, and $b>1$, we have

$$
\begin{gathered}
\mathrm{P}\left(\tau_{k+1}-\tau_{k}>\left|v_{k}\right|^{-1+\delta} \text { for some } k \text { such that } \tau_{n}<\tau_{k}<\bar{\tau}_{n} \mid \mathcal{G}_{n}\right) \leq r^{-N} \\
\mathrm{P}\left(\sup _{\tau_{k} \leq t \leq \tau_{k+1}}\left|\dot{Y}(t)-v_{k}\right|>\left|v_{k}\right|^{-1+\delta} \text { for some } k \text { such that } \tau_{n} \leq \tau_{k}<\bar{\tau}_{n} \mid \mathcal{G}_{n}\right) \leq r^{-N}, \\
\mathrm{P}\left(\bar{\tau}_{n}=\infty \mid \mathcal{G}_{n}\right) \leq r^{-N}
\end{gathered}
$$

for all sufficiently large $r$ almost surely on the event $\left|v_{n}\right| \in(r, b r)$. 
Lemma 5.6. Assume that $v_{0}=\left(\left|v_{0}\right|, 0, \ldots, 0\right)$, and $\left|v_{0}\right| \rightarrow \infty$. Then both families of processes $\dot{Y}\left(\left|v_{0}\right|^{3} t\right) /\left|v_{0}\right|$ and $\dot{Z}\left(\left|v_{0}\right|^{3} t\right) /\left|v_{0}\right|$ converge weakly to the diffusion process $\bar{V}(t)$ given by (7) starting at $(1,0, \ldots, 0)$.

This lemma is a slight modification of the results of [4, 5, 6] to the case of the processes $Y(t)$ and $Z(t)$, so we omit the proof. For example, the main theorem of [5] on page 24 gives the desired result, except the fact that in the setting of [5] there is no renewal of the force field. The proof, however, goes through without major modifications.

Corollary 5.7. For each $a>0$ and $b>1$ there is $c<1$ such that

$$
\mathrm{P}\left(\hat{\tau}_{0}^{a}<\check{\tau}_{0}^{b}\right) \leq c
$$

for all sufficiently large $r$ and all $\left|v_{0}\right| \in(r, b r)$. The same is true if $\hat{\tau}_{0}^{a}$ and $\check{\tau}_{0}^{b}$ are defined as the stopping times for the process $Z(t)$ with initial velocity $v_{0}$.

Proof. It is sufficient to consider the process $Y(t)$ since the proof for the process $Z(t)$ is completely similar. From Lemma 5.6 and the rotation-invariance of the force field it follows that when $\left|v_{0}\right| \rightarrow \infty$, the processes $\left|\dot{Y}\left(\left|v_{0}\right|^{3} t\right)\right| /\left|v_{0}\right|$ converge weakly to the diffusion process $|\bar{V}(t)|$ with $|\bar{V}(0)|=1$. Therefore

$$
\begin{gathered}
\limsup _{\left|v_{0}\right| \rightarrow \infty} \mathrm{P}\left(\frac{\left|v_{0}\right|}{b}<|\dot{Y}(t)|<\left|v_{0}\right| b \text { for all } 0 \leq t \leq a\left|v_{0}\right|^{3}\right)= \\
\limsup _{\left|v_{0}\right| \rightarrow \infty} \mathrm{P}\left(\frac{1}{b}<\left|\dot{Y}\left(\left|v_{0}\right|^{3} t\right)\right| /\left|v_{0}\right|<b \text { for all } 0 \leq t \leq a\right) \leq \\
\mathrm{P}\left(\frac{1}{2 b}<|\bar{V}(t)|<2 b \text { for all } 0 \leq t \leq a\right)<1,
\end{gathered}
$$

where the first inequality holds due to the weak convergence of $\dot{Y}\left(\left|v_{0}\right|^{3} t\right)|/| v_{0} \mid$ to $\bar{V}(t)$ since the closure of the set $\{\varphi \in C([0, a], \mathbb{R}): 1 / b<\varphi(t)<b$ for all $0 \leq t \leq a\}$ is contained in the open set $\{\varphi \in C([0, a], \mathbb{R}): 1 / 2 b<\varphi(t)<2 b$ for all $0 \leq t \leq a\}$. The second inequality is due to the fact that $|\bar{V}(t)|$ is a non-degenerate diffusion process on $(0, \infty)$ starting at 1 , as follows from (77) and Lemma 5.6. The Corollary now follows from the definitions of $\hat{\tau}_{0}^{a}$ and $\check{\tau}_{0}^{b}$.

Now we can replace the stopping time $\bar{\tau}_{0}$ by $\check{\tau}_{0}^{b}$ in Lemma 5.4 .

Lemma 5.8. For each $N, \delta>0$, and $b>1$, we have

$$
\begin{gathered}
\mathrm{P}\left(\tau_{n+1}-\tau_{n}>\left|v_{n}\right|^{-1+\delta} \text { for some } n \text { such that } \tau_{n}<\check{\tau}_{0}^{b}\right) \leq r^{-N}, \\
\mathrm{P}\left(\sup _{\tau_{n} \leq t \leq \tau_{n+1}}\left|\dot{Y}(t)-v_{n}\right|>\left|v_{n}\right|^{-1+\delta} \text { for some } n \text { such that } \tau_{n}<\check{\tau}_{0}^{b}\right) \leq r^{-N}, \\
\mathrm{P}\left(\check{\tau}_{0}^{b}=\infty\right) \leq r^{-N}
\end{gathered}
$$

for all sufficiently large $r$ and all $\left|v_{0}\right| \in(r, b r)$. 
Proof. Let

$$
q_{Y}=q_{Y}(r)=\sup _{v_{0}:\left|v_{0}\right| \in(r, b r)} \mathrm{P}\left(\tau_{n+1}-\tau_{n}>\left|v_{n}\right|^{-1+\delta} \text { for some } n \text { such that } \tau_{n}<\check{\tau}_{0}^{b}\right) .
$$

Let $q_{Z}=q_{Z}(r)$ be defined as $q_{Y}$, with the only difference that the stopping times are assumed to correspond to the process $Z(t)$ instead of $Y(t)$. Take an arbitrary $a>0$. Then, for $\left|v_{0}\right| \in(r, b r)$ we have

$$
\begin{gathered}
\mathrm{P}\left(\tau_{n+1}-\tau_{n}>\left|v_{n}\right|^{-1+\delta} \text { for some } n \text { such that } \tau_{n}<\check{\tau}_{0}^{b}\right) \leq \\
\mathrm{P}\left(\tau_{n+1}-\tau_{n}>\left|v_{n}\right|^{-1+\delta} \text { for some } n \text { such that } \tau_{n}<\bar{\tau}_{0}\right)+ \\
\mathrm{P}\left(\tau_{n+1}-\tau_{n}>\left|v_{n}\right|^{-1+\delta} \text { for some } n \text { such that } \bar{\tau}_{0} \leq \tau_{n}<\check{\tau}_{0}^{b}\right) .
\end{gathered}
$$

The first term in the right-hand side does not exceed $r^{-N}$ by Lemma 5.4. In order to estimate the second term, we observe that

$$
\begin{gathered}
\mathrm{P}\left(\tau_{n+1}-\tau_{n}>\left|v_{n}\right|^{-1+\delta} \text { for some } n \text { such that } \bar{\tau}_{0} \leq \tau_{n}<\check{\tau}_{0}^{b}\right)= \\
\mathrm{P}\left(\hat{\tau}_{0}^{a}<\check{\tau}_{0}^{b} \text { and } \tau_{n+1}-\tau_{n}>\left|v_{n}\right|^{-1+\delta} \text { for some } n \text { such that } \hat{\tau}_{0}^{a} \leq \tau_{n}<\check{\tau}_{0}^{b}\right) \leq \\
\mathrm{P}\left(\hat{\tau}_{0}^{a}<\check{\tau}_{0}^{b}\right) q_{Z},
\end{gathered}
$$

where the inequality is due to the Markov property with respect to the stopping time $\hat{\tau}_{0}^{a}$ (see formula (15)). Therefore, by Corollary [5.7,

$$
q_{Y} \leq r^{-N}+c q_{Z} .
$$

Similarly,

$$
q_{Z} \leq r^{-N}+c q_{Z}
$$

Since $c<1$ and $N$ is arbitrary, these two inequalities imply (38). The proof of (391) is similar. In order to prove (401), define

$$
\bar{q}_{Y}(k)=\sup _{v_{0}:\left|v_{0}\right| \in(r, b r)} \mathrm{P}\left(\check{\tau}_{0}^{b}>2 k a r^{3}\right), \quad k \geq 0 .
$$

Let $\bar{q}_{Z}(k)$ be defined as $\bar{q}_{Y}(k)$, with the only difference that the stopping times are assumed to correspond to the process $Z(t)$ instead of $Y(t)$. Note that for $\left|v_{0}\right| \in(r, b r)$ we have

$$
\mathrm{P}\left(\check{\tau}_{0}^{b}>2 k a r^{3}\right) \leq \mathrm{P}\left(\hat{\tau}_{0}^{a}>2 a r^{3}, \check{\tau}_{0}^{b}>2 k a r^{3}\right)+\mathrm{P}\left(\hat{\tau}_{0}^{a} \leq 2 a r^{3}, \check{\tau}_{0}^{b}>2 k a r^{3}\right), k \geq 1 .
$$

Note that if $\hat{\tau}_{0}^{a}, \breve{\tau}_{0}^{b}>2 a r^{3}$, then $\tau_{k+1}-\tau_{k}>a r^{3}$ for some $k$ with $\tau_{k}<\bar{\tau}_{0}$. Therefore, the first term on the right-hand side can be estimated from above by $r^{-N}$ due to (35). By the Markov property with respect to the stopping time $\hat{\tau}_{0}^{a}$ (see formula (15)), the second term does not exceed $\mathrm{P}\left(\hat{\tau}_{0}^{a}<\check{\tau}_{0}^{b}\right) \bar{q}_{Z}(k-1) \leq c \bar{q}_{Z}(k-1)$, where $c$ is the constant from Corollary 5.7. Therefore,

$$
\bar{q}_{Y}(k) \leq r^{-N}+c \bar{q}_{Z}(k-1) .
$$


Similarly,

$$
\bar{q}_{Z}(k) \leq r^{-N}+c \bar{q}_{Z}(k-1) .
$$

Since $c<1$ and $N$ is arbitrary, these two inequalities imply that

$$
\max \left(\bar{q}_{Y}(k), \bar{q}_{Z}(k)\right) \leq r^{-N}+c^{k} .
$$

This implies (40) since an arbitrarily large $k$ can be taken.

Corollary 5.9. For each $N, \delta>0$, and $b>1$, we have

$$
\begin{gathered}
\mathrm{P}\left(\tau_{k+1}-\tau_{k}>\left|v_{k}\right|^{-1+\delta} \text { for some } k \text { such that } \tau_{n}<\tau_{k}<\check{\tau}_{n}^{b} \mid \mathcal{G}_{n}\right) \leq r^{-N}, \\
\mathrm{P}\left(\sup _{\tau_{k} \leq t \leq \tau_{k+1}}\left|\dot{Y}(t)-v_{k}\right|>\left|v_{k}\right|^{-1+\delta} \text { for some } k \text { such that } \tau_{n} \leq \tau_{k}<\check{\tau}_{n}^{b} \mid \mathcal{G}_{n}\right) \leq r^{-N}, \\
\mathrm{P}\left(\check{\tau}_{n}^{b}=\infty \mid \mathcal{G}_{n}\right) \leq r^{-N}
\end{gathered}
$$

for all sufficiently large $r$ almost surely on each of the events $\left|v_{n}\right| \in(r, b r)$.

Lemma 5.10. For each $N, \delta>0$, and $k>0$ we have

$$
\mathrm{P}\left(\sup _{0 \leq t \leq\left|v_{0}\right|^{3-\delta}}\left|\dot{Y}(t)-v_{0}\right|>k\left|v_{0}\right|\right) \leq\left|v_{0}\right|^{-N}
$$

for all sufficiently large $\left|v_{0}\right|$.

Proof. Let us write

$$
\dot{Y}(t)-v_{0}=\left(v_{1}-v_{0}\right)+\left(v_{2}-v_{1}\right)+\ldots+\left(v_{n}-v_{n-1}\right)+\dot{Y}(t)-v_{n},
$$

where $n=n(t)$ is the random time such that $\tau_{n-1} \leq t<\tau_{n}$. Without loss of generality we may assume that $k \leq 1 / 2$. Let $L=L\left(v_{0}\right)=\left[2\left(\left|v_{0}\right|^{4-\delta} l^{-1}+1\right)\right]$, where $l=4 R+1$ is the constant used in the definition of the stopping times $\tau_{n}$. Let $\sigma$ be the random time defined by

$$
\sigma=\min \left\{m:\left|\left(v_{1}-v_{0}\right)+\left(v_{2}-v_{1}\right)+\ldots+\left(v_{m}-v_{m-1}\right)\right| \geq k\left|v_{0}\right| / 2\right\} \wedge L .
$$

Observe that if $|\dot{Y}(t)|$ does not exceed $2\left|v_{0}\right|$ on the time interval $\left[0,\left|v_{0}\right|^{3-\delta}\right]$, then $\tau_{L} \geq$ $\left|v_{0}\right|^{3-\delta}$ since $\tau_{n+1}-\tau_{n} \geq l /\left(2\left|v_{0}\right|\right)$ for each $n$ such that $\tau_{n}<\left|v_{0}\right|^{3-\delta}$, as follows from the definition of the stopping times $\tau_{n}$. Therefore,

$$
\begin{gathered}
\left\{\sup _{0 \leq t \leq\left|v_{0}\right|^{3-\delta}}\left|\dot{Y}(t)-v_{0}\right|>k\left|v_{0}\right|\right\} \subseteq \\
\subseteq\{\sigma<L\} \cup\left(\{\sigma=L\} \cap\left\{\sup _{0 \leq t \leq\left|v_{0}\right|^{3-\delta}}\left|\dot{Y}(t)-v_{0}\right|>k\left|v_{0}\right|\right\}\right)
\end{gathered}
$$




$$
\begin{gathered}
\subseteq\{\sigma<L\} \cup\left(\{\sigma=L\} \cap \bigcup_{m=1}^{L}\left\{\sup _{\tau_{m-1} \leq t \leq \tau_{m}}\left|\dot{Y}(t)-v_{0}\right| \geq k\left|v_{0}\right|\right\}\right) \\
\subseteq\{\sigma<L\} \cup\left(\{\sigma=L\} \cap \bigcup_{m=1}^{L}\left\{\sup _{\tau_{m-1} \leq t \leq \tau_{m}}\left|\dot{Y}(t)-v_{m-1}\right| \geq k\left|v_{0}\right| / 2\right\}\right) .
\end{gathered}
$$

Define $c_{i}, i \geq 1$, by

$$
\left(v_{i}-v_{i-1}\right)=\int_{\tau_{i-1}}^{\eta_{i}} \widetilde{F}_{i-1}\left(z_{i-1}(s)\right) d s+c_{i} .
$$

By Corollary 5.2 and Lemma 5.3 (formulas (31), (32), and (34)), for each $N$ and $\varepsilon>0$ the estimates

$$
\begin{gathered}
\mathrm{P}\left(\left|c_{i}\right|>\left|v_{0}\right|^{-1+\varepsilon} \mid \mathcal{G}_{i-1}\right) \leq\left|v_{0}\right|^{-N}, \\
\mathrm{E}\left(\left|c_{i}\right| \chi_{\left\{\left|c_{i}\right| \leq\left|v_{0}\right|^{-1+\varepsilon}\right\}} \mid \mathcal{G}_{i-1}\right) \leq\left|v_{0}\right|^{-3+\varepsilon}
\end{gathered}
$$

hold for each $i$ on $\{\sigma \geq i\}$ if $\left|v_{0}\right|$ is sufficiently large. Let

$$
\begin{gathered}
C_{j}=\sum_{i=1}^{j \wedge \sigma}\left|c_{i}\right|, \\
h_{j}=\sum_{i=1}^{j \wedge \sigma}\left(\left|c_{i}\right| \chi_{\left\{\left|c_{i}\right| \leq\left|v_{0}\right|^{-1+\varepsilon}\right\}}-\left|v_{0}\right|^{-3+\varepsilon}\right) .
\end{gathered}
$$

By (44) and (45), $h_{j}$ is a supermartingale. Let $h_{j}=\alpha_{j}+\beta_{j}$ be the Doob decomposition of $h_{j}$, where

$$
\beta_{j}=\sum_{i=1}^{j \wedge \sigma} \mathrm{E}\left(\left(\left|c_{i}\right| \chi_{\left\{\left|c_{i}\right| \leq\left|v_{0}\right|^{-1+\varepsilon}\right\}}-\left|v_{0}\right|^{-3+\varepsilon}\right) \mid \mathcal{G}_{i-1}\right)
$$

is a non-increasing process. Let $\langle\alpha\rangle_{j}$ be the quadratic variation of $\alpha_{j}$. It is equal to

$$
\langle\alpha\rangle_{j}=\sum_{i=1}^{j} \mathrm{E}\left(\left(\left(h_{i}-h_{i-1}\right)-\left(\beta_{i}-\beta_{i-1}\right)\right)^{2} \mid \mathcal{G}_{i-1}\right), \quad j \geq 1 .
$$

From (46) it follows that $\left|h_{i}-h_{i-1}\right| \leq 2\left|v_{0}\right|^{-1+\varepsilon}$ for sufficiently large $\left|v_{0}\right|$, and consequently $\left|\beta_{i}-\beta_{i-1}\right| \leq 2\left|v_{0}\right|^{-1+\varepsilon}$. Therefore $\langle\alpha\rangle_{j} \leq 16 j\left|v_{0}\right|^{-2+2 \varepsilon}$, which implies that for each $p \in \mathbb{N}$ there is a constant $k_{p}$ such that

$$
\langle\alpha\rangle_{j}^{p} \leq k_{p}\left(j\left|v_{0}\right|^{-2+2 \varepsilon}\right)^{p} .
$$

Applying this inequality to $j=L$ and noting that $\sigma \leq L$ and $\langle\alpha\rangle_{j}^{p}$ is non-decreasing in $j$, we obtain

$$
\langle\alpha\rangle_{\sigma}^{p} \leq k_{p}^{\prime}\left(\left|v_{0}\right|^{2+2 \varepsilon-\delta}\right)^{p} .
$$


Take $\varepsilon=\delta / 3$. Then, by the Chebyshev Inequality and the Martingale Moment Inequality, for each $N$ there are $p$ and $K_{p}$ such that

$$
\begin{gathered}
\mathrm{P}\left(h_{\sigma} \geq k\left|v_{0}\right| / 8\right) \leq \mathrm{P}\left(\alpha_{\sigma} \geq k\left|v_{0}\right| / 8\right) \leq \mathrm{P}\left(\left|\alpha_{\sigma}\right|^{2 p} \geq\left(k\left|v_{0}\right| / 8\right)^{2 p}\right) \leq \\
\frac{\mathrm{E}\left|\alpha_{\sigma}\right|^{2 p}}{\left(k\left|v_{0}\right| / 8\right)^{2 p}} \leq \frac{K_{p} \mathrm{E}\langle\alpha\rangle_{\sigma}^{p}}{\left(k\left|v_{0}\right| / 8\right)^{2 p}} \leq\left|v_{0}\right|^{-N}
\end{gathered}
$$

if $\left|v_{0}\right|$ is sufficiently large. Note that

$$
\begin{gathered}
\mathrm{P}\left(C_{\sigma} \geq k\left|v_{0}\right| / 4\right) \leq \\
\mathrm{P}\left(h_{\sigma} \geq k\left|v_{0}\right| / 8\right)+\mathrm{P}\left(C_{\sigma}-h_{\sigma} \geq k\left|v_{0}\right| / 8\right) \leq \\
\left|v_{0}\right|^{-N}+\mathrm{P}\left(\sum_{i=1}^{\sigma}\left(\left|c_{i}\right| \chi_{\left\{\left|c_{i}\right|>\left|v_{0}\right|^{-1+\varepsilon}\right\}}+\left|v_{0}\right|^{-3+\varepsilon}\right) \geq k\left|v_{0}\right| / 8\right) .
\end{gathered}
$$

Since $\sigma \leq L$, and therefore $\sigma\left|v_{0}\right|^{-3+\varepsilon}<k\left|v_{0}\right| / 8$ for all sufficiently large $\left|v_{0}\right|$, the second term in the right hand side of (47) is estimated from above by

$$
\mathrm{P}\left(\left|c_{i}\right|>\left|v_{0}\right|^{-1+\varepsilon} \text { for some } 1 \leq i \leq \sigma\right) \leq L\left|v_{0}\right|^{-N}
$$

where the inequality follows after integrating both sides of (44) over the event $\{\sigma \geq i\}$ and recalling that $\sigma \leq L$. Since $N$ was arbitrary and $L \leq\left|v_{0}\right|^{4}$ for all sufficiently large $\left|v_{0}\right|$, this implies that

$$
\mathrm{P}\left(C_{\sigma} \geq k\left|v_{0}\right| / 4\right) \leq\left|v_{0}\right|^{-N}
$$

if $\left|v_{0}\right|$ is sufficiently large. Let

$$
f_{j}=\sum_{i=1}^{j \wedge \sigma} \int_{\tau_{i-1}}^{\eta_{i}} \widetilde{F}_{i-1}\left(z_{i-1}(s)\right) d s, \quad j \geq 1
$$

Notice that $f_{j}$ is a martingale vector. Indeed,

$$
\mathrm{E}\left(f_{j}-f_{j-1} \mid \mathcal{G}_{i-1}\right)=\chi_{\{j \leq \sigma\}} \mathrm{E}\left(\int_{\tau_{j-1}}^{\eta_{j}} \widetilde{F}_{j-1}\left(z_{j-1}(s)\right) d s \mid \mathcal{G}_{i-1}\right) .
$$

Let $\widetilde{\mathcal{G}}_{n}$ be the $\sigma$-algebra determined by the Poisson field $r_{i}^{n}$ (see the definition of the random field $F_{n}$ in Section 4.1). Then the last conditional expectation can be written as

$$
\mathrm{E}\left(\int_{\tau_{j-1}}^{\eta_{j}} \widetilde{F}_{j-1}\left(z_{j-1}(s)\right) d s \mid \mathcal{G}_{i-1}\right)=\mathrm{E}\left(\mathrm{E}\left(\int_{\tau_{j-1}}^{\eta_{j}} \widetilde{F}_{j-1}\left(z_{j-1}(s)\right) d s \mid \sigma\left(\mathcal{G}_{i-1} \cup \widetilde{\mathcal{G}}_{i-1}\right)\right) \mid \mathcal{G}_{i-1}\right)
$$

The inner conditional expectation is equal to zero since the functions $f_{i}^{n}$ from the definition of the fields $\widetilde{F}_{n}$ are independent of the Poisson fields, and are symmetrically distributed by (5). 
We shall denote the components of the vector $f_{j}$ by $f_{j}^{a}, 1 \leq a \leq d$. The quadratic variation of $f_{j}$ is

$$
\left\langle f^{a}, f^{b}\right\rangle_{j}=\sum_{i=1}^{j \wedge \sigma} \mathrm{E}\left(\left(\int_{\tau_{i-1}}^{\eta_{i}} \widetilde{F}_{i-1}^{a}\left(z_{i-1}(s)\right) d s\right)\left(\int_{\tau_{i-1}}^{\eta_{i}} \widetilde{F}_{i-1}^{b}\left(z_{i-1}(s)\right) d s\right) \mid \mathcal{G}_{i-1}\right), \quad j \geq 1 .
$$

Using arguments similar to those in the proof of Lemma 5.1 (see the justification of formula (22) $)$, it is easy to show that for $p \in \mathbb{N}$ there is a constant $k_{p}^{\prime \prime}$ such that for all $j \leq L$ we have

$$
\mathrm{E}\left|\langle f\rangle_{j}\right|^{p} \leq k_{p}^{\prime \prime}\left(j\left|v_{0}\right|^{-2+\delta / 2}\right)^{p}
$$

if $\left|v_{0}\right|$ is sufficiently large, where $\left|\langle f\rangle_{j}\right|$ stands for the norm of the matrix $\left\langle f^{a}, f^{b}\right\rangle_{j}$. In particular, for $j=L$ we obtain

$$
\mathrm{E}\left|\langle f\rangle_{\sigma}\right|^{p} \leq k_{p}^{\prime \prime \prime}\left(\left|v_{0}\right|^{2-\delta / 2}\right)^{p}
$$

By the Chebyshev Inequality and the Martingale Moment Inequality, for each $N$ there are $p \in \mathbb{N}$ and $K_{p}>0$ such that

$$
\mathrm{P}\left(\left|f_{\sigma}\right| \geq k\left|v_{0}\right| / 4\right)=\mathrm{P}\left(\left|f_{\sigma}\right|^{2 p} \geq\left(k\left|v_{0}\right| / 4\right)^{2 p}\right) \leq \frac{\mathrm{E}\left|f_{\sigma}\right|^{2 p}}{\left(k\left|v_{0}\right| / 4\right)^{2 p}} \leq \frac{K_{p} \mathrm{E}\left|\langle f\rangle_{\sigma}\right|^{p}}{\left(k\left|v_{0}\right| / 4\right)^{2 p}} \leq\left|v_{0}\right|^{-N}
$$

if $\left|v_{0}\right|$ is sufficiently large.

Together with (48), this implies that

$$
\mathrm{P}(\sigma<L) \leq\left|v_{0}\right|^{-N}
$$

It easily follows from Corollary 5.2 that

$$
\mathrm{P}\left(\{\sigma=L\} \cap \bigcup_{m=1}^{L}\left\{\sup _{\tau_{m-1} \leq t \leq \tau_{m}}\left|\dot{Y}(t)-v_{m-1}\right| \geq k\left|v_{0}\right| / 2\right\}\right) \leq\left|v_{0}\right|^{-N}
$$

\section{Long time behavior of $Y(t)$.}

The goal of this section is to show that the paths of $Y(t)$ are not self-intersecting with probability close to one, and therefore the distributions of $X$ and $Y$ are close, as claimed. This is achieved in subsection 6.2. In subsection 6.1 we establish some a priori bounds on the growth of $|\dot{Y}|$. 


\subsection{Behavior of $|\dot{Y}(t)|$ as $t \rightarrow \infty$.}

In this section we shall demonstrate that for large $\left|v_{0}\right|$ with high probability the norm of the velocity vector $|\dot{Y}(t)|$ grows as $t^{1 / 3}$ when $t \rightarrow \infty$.

The idea of the proof is the following. We consider $\dot{Y}$ at the moments $s_{n}$ its modulus crosses $2^{l}$ (alternating odd and even $l$ ). By Lemma 5.6 and (12), $\ln \left|\dot{Y}\left(s_{n}\right)\right|$ can be well approximated by a simple random walk biased to the right. It follows that $\left|\dot{Y}\left(s_{n}\right)\right|$ grows exponentially and so $|\dot{Y}(t)|$ spends most of the time near its maximum. By Lemma 5.6, $s_{n+1}-s_{n}$ is of order $\left|\dot{Y}\left(s_{n}\right)\right|^{3}$, which implies the desired result. Let us now give a detailed proof.

We start by describing a discretized version of the process $|\dot{Y}(t)|$. Let $2^{m-\frac{1}{2}} \leq\left|v_{0}\right|<$ $2^{m+\frac{1}{2}}$ for some $m \in \mathbb{Z}$. Let $0<\delta<1$. Define, inductively, a sequence of events $\mathcal{E}_{n}^{\delta}$ and three processes $s_{n}, t_{n} \in \mathbb{R}^{+} \cup \infty$ and $\xi_{n} \in \mathbb{Z}$ as follows. Let $\mathcal{E}_{0}^{\delta}=\Omega, s_{0}=t_{0}=0$, and $\xi_{0}=m$. Assume that $\mathcal{E}_{n-1}^{\delta}, s_{n-1}, t_{n-1}$, and $\xi_{n-1}$ have been defined for some $n \geq 1$. We then define

$$
\begin{gathered}
s_{n}=\inf \left\{t:|\dot{Y}(t)|=2^{\xi_{n-1}-1} \text { or }|\dot{Y}(t)|=2^{\xi_{n-1}+1}\right\}, \\
t_{n}=\min \left\{\tau_{k}: \tau_{k} \geq s_{n}\right\}, \text { and } \xi_{n}=\log _{2}\left|\dot{Y}\left(s_{n}\right)\right| . \\
\mathcal{E}_{n}^{\delta}=\mathcal{E}_{n-1}^{\delta} \cap\left\{t_{n}<\infty\right\} \cap\left\{\tau_{k+1}-\tau_{k} \leq\left|v_{k}\right|^{-1+\delta} \text { for all } k \text { such that } t_{n-1}<\tau_{k} \leq t_{n}\right\} \cap \\
\left.\cap \sup _{\tau_{k} \leq t \leq \tau_{k+1}}\left|\dot{Y}(t)-v_{k}\right| \leq\left|v_{k}\right|^{-1+\delta} \text { for all } k \text { such that } t_{n-1} \leq \tau_{k} \leq t_{n}\right\} .
\end{gathered}
$$

Let $\mathcal{F}_{n}$ be the $\sigma$-algebra of events determined before $t_{n}$, that is $\mathcal{F}_{n}=\sigma\left(\cup_{m: \tau_{m} \leq t_{n}} \mathcal{G}_{m}\right)$. The process $\xi_{n}$ can be viewed as a random walk (with memory and random transition times), while $t_{n}$ can be viewed as transition times for the random walk. Note that the process $|\dot{Y}(t)|$ takes values equal to powers of 2 at times $s_{n}$. It is more convenient, however, to consider times $t_{n}$ (which are close to times $s_{n}$, but coincide with the stopping times $\tau_{k}$ ), and the $\sigma$-algebras $\mathcal{F}_{n}$ are defined using the times $t_{n}$.

The following lemma describes the one-step transition times and transition probabilities.

Lemma 6.1. (a) $\mathcal{E}_{n}^{\delta}$ is $\mathcal{F}_{n}$-measurable. For each $N>0$ there is $M$ such that for $m \geq M$ we have

$$
\mathrm{P}\left(\mathcal{E}_{n}^{\delta} \mid \mathcal{F}_{n-1}\right) \geq 1-2^{-N m} \quad \text { almost surely on }\left\{\xi_{n-1}=m\right\} \cap \mathcal{E}_{n-1}^{\delta} .
$$

(b) For each $N>0$ there exist $M$ and $0<c<1$, such that for $m \geq M$ we have

$$
\mathrm{P}\left(t_{n}-t_{n-1}>2^{3 m} k \mid \mathcal{F}_{n-1}\right) \leq c^{k}+2^{-N m}, \quad k \geq 1 \text {, almost surely on }\left\{\xi_{n-1}=m\right\} \cap \mathcal{E}_{n-1}^{\delta} .
$$

(c) There exist $M$ and $0<c<1$, such that for $m \geq M$ and $n \geq 2$ we have

$$
\mathrm{P}\left(t_{n}-t_{n-1}<2^{3 m} \mid \mathcal{F}_{n-1}\right) \leq c \text { almost surely on }\left\{\xi_{n-1}=m\right\} \cap \mathcal{E}_{n-1}^{\delta} .
$$

(d) There is $p>1 / 2$ such that for each $\varepsilon>0$ there exists $M$, such that for $m \geq M$ and $n \geq 2$ we have

$$
\left|\mathrm{P}\left(\xi_{n}=\xi_{n-1}+1 \mid \mathcal{F}_{n-1}\right)-p\right| \leq \varepsilon \text { almost surely on }\left\{\xi_{n-1}=m\right\} \cap \mathcal{E}_{n-1}^{\delta} .
$$


Proof. (a) The fact that $\mathcal{E}_{n}^{\delta}$ is $\mathcal{F}_{n}$-measurable follows from the definition of $\mathcal{E}_{n}^{\delta}$ and $\mathcal{F}_{n}$.

Next, observe that if $\mathcal{E}_{n-1}^{\delta}$ happens, then to ensure that $\mathcal{E}_{n}^{\delta}$ happens we need to exclude three events: $\left\{t_{n}=\infty\right\},\left\{\tau_{k+1}-\tau_{k}>\left|v_{k}\right|^{-1+\delta}\right.$ for some $\left.t_{n-1} \leq \tau_{k} \leq t_{n}\right\}$ and

$$
\left\{\sup _{\tau_{k} \leq t \leq \tau_{k+1}}\left|\dot{Y}-v_{k}\right|>\left|v_{k}\right|^{-1+\delta} \text { for some } t_{n-1} \leq \tau_{k} \leq t_{n}\right\}
$$

Therefore (50) follows from Corollary [5.9 with $b=4$ and $r=2^{m-1}$.

(b) The statement follows from (42) once we notice that $\left|\dot{Y}\left(t_{n-1}\right)\right| \in\left(2^{m-1}, 2^{m+1}\right)$ on $\left\{\xi_{n-1}=m\right\} \cap \mathcal{E}_{n-1}^{\delta}$ if $m$ is sufficiently large.

(c) This follows from Lemma 5.6 once we take into account that, by the definition of $\mathcal{E}_{n-1}^{\delta}$, for $n \geq 2$ and all sufficiently large $m$ we have

$$
|| \dot{Y}\left(t_{n-1}\right)\left|-2^{m}\right| \leq 1 \text { on }\left\{\xi_{n-1}=m\right\} \cap \mathcal{E}_{n-1}^{\delta} .
$$

(d) Consider the limiting process $\bar{V}(t)$ with $|\bar{V}(0)|=1$. Let $p$ be the probability that the process $|\bar{V}(t)|$ reaches 2 before reaching $1 / 2$. Notice that $p>1 / 2$. Therefore, the statement follows from Lemma 5.6 and (51).

Lemma 6.2. For $\delta>0$ we have

$$
\lim _{\left|v_{0}\right| \rightarrow \infty} \mathrm{P}\left(\left(\left|v_{0}\right|+t^{1 / 3}\right)^{1-\delta} \leq|\dot{Y}(t)| \leq\left(\left|v_{0}\right|+t^{1 / 3}\right)^{1+\delta} \quad \text { for all } t \geq 0\right)=1 \text {. }
$$

Proof. Let $\bar{\varepsilon}$ be fixed and $\varepsilon$ be a positive constant, to be specified later. Let $A_{v_{0}}$ be the following event

$$
A_{v_{0}}=\left(\bigcap_{n=0}^{\infty} \mathcal{E}_{n}^{\varepsilon}\right) \cap\left\{\left|\xi_{n}-p n-\xi_{0}\right| \leq \varepsilon\left(n+\xi_{0}\right) \text { for all } n\right\}
$$

( $p$ is the constant from Lemma 6.1(d)).

From parts (a) and (d) of Lemma 6.1 it easily follows that we can take a large enough $M$ such that

$$
\mathrm{P}\left(A_{v_{0}}\right) \geq 1-\bar{\varepsilon} / 3
$$

if $v_{0}$ is such that $\xi_{0}>M$. By part (b) of Lemma 6.1,

$$
\mathrm{P}\left(A_{v_{0}} \cap\left\{t_{n}-t_{n-1} \geq k(n) 2^{3\left(p(n-1)+\xi_{0}+\varepsilon\left(n-1+\xi_{0}\right)\right)}\right\}\right) \leq c^{k(n)}+2^{-N\left(p(n-1)+\xi_{0}-\varepsilon\left(n-1+\xi_{0}\right)\right)}
$$

for each $n$, where $0<c<1$. Take $k(n)=2^{\varepsilon\left(n+\xi_{0}\right)}$. Let

$$
B_{v_{0}}=\left\{t_{n}-t_{n-1} \geq 2^{\varepsilon\left(n+\xi_{0}\right)} 2^{3\left(p(n-1)+\xi_{0}+\varepsilon\left(n-1+\xi_{0}\right)\right)} \text { for some } n\right\} .
$$

Then

$$
\mathrm{P}\left(A_{v_{0}} \cap B_{v_{0}}\right) \leq \sum_{n=1}^{\infty}\left(c^{2^{\varepsilon\left(n+\xi_{0}\right)}}+2^{-N\left(p(n-1)+\xi_{0}-\varepsilon\left(n-1+\xi_{0}\right)\right)}\right)
$$


The right-hand side of this inequality can be made smaller than $\bar{\varepsilon} / 3$ by taking sufficiently large $M$.

Notice that for each $a(n)$ and $\bar{k}(n)$

$$
A_{v_{0}} \cap\left\{t_{n}<a(n)\right\} \subseteq A_{v_{0}} \cap\left\{t_{n}-t_{n-1}<a(n)\right\} \cap \ldots \cap\left\{t_{n-\bar{k}(n)}-t_{n-\bar{k}(n)-1}<a(n)\right\} .
$$

Let $\bar{k}(n)=\varepsilon\left(n+\xi_{0}\right)$ and $a(n)=2^{3\left(p(n-1-\bar{k}(n))+\xi_{0}-\varepsilon\left(n-1+\xi_{0}\right)\right)}$. By part (c) of Lemma 6.1, the probability of the event in the right-hand side of (53) is estimated from above by $c^{\bar{k}(n)+1}$, where $0<c<1$. Let

$$
C_{v_{0}}=\left\{t_{n}<2^{3\left(p\left(n-1-\varepsilon\left(n+\xi_{0}\right)\right)+\xi_{0}-\varepsilon\left(n-1+\xi_{0}\right)\right)} \text { for some } n\right\} .
$$

Then

$$
\mathrm{P}\left(A_{v_{0}} \cap C_{v_{0}}\right) \leq \sum_{n=1}^{\infty} c^{\varepsilon\left(n+\xi_{0}\right)+1} .
$$

The right-hand side of this inequality can be made smaller than $\bar{\varepsilon} / 3$ by taking sufficiently large $M$. We have thus obtained that

$$
\mathrm{P}\left(A_{v_{0}} \backslash\left(B_{v_{0}} \cup C_{v_{0}}\right)\right) \geq 1-\bar{\varepsilon}
$$

On the event $A_{v_{0}} \backslash\left(B_{v_{0}} \cup C_{v_{0}}\right)$ we have

$$
\begin{gathered}
\left|\xi_{n}-p n-\xi_{0}\right| \leq \varepsilon\left(n+\xi_{0}\right) \quad \text { for all } n \\
t_{n}-t_{n-1} \leq 2^{3\left(p(n-1)+\xi_{0}+\varepsilon\left(n-1+\xi_{0}\right)\right)+\varepsilon\left(n+\xi_{0}\right)} \text { for all } n \\
t_{n} \geq 2^{3\left(p\left(n-1-\varepsilon\left(n+\xi_{0}\right)\right)+\xi_{0}-\varepsilon\left(n-1+\xi_{0}\right)\right)} \text { for all } n
\end{gathered}
$$

Since $\varepsilon$ can be taken arbitrarily small, these three inequalities imply that for each $\delta>0$

$$
\left(2^{\xi_{0}}+t_{n}^{\frac{1}{3}}\right)^{1-\delta} \leq 2^{\xi_{n}} \leq\left(2^{\xi_{0}}+t_{n}^{\frac{1}{3}}\right)^{1+\delta} \text { for all } n \geq 0
$$

on $A_{v_{0}} \backslash\left(B_{v_{0}} \cup C_{v_{0}}\right)$, provided that $M$ is sufficiently large. This implies the statement of the lemma since $2^{\xi_{n}-2} \leq|\dot{Y}(t)| \leq 2^{\xi_{n}+2}$ for $t_{n} \leq t \leq t_{n+1}$ on $A_{v_{0}} \backslash\left(B_{v_{0}} \cup C_{v_{0}}\right)$ due to (51).

Corollary 6.3. For $\delta>0$ we have

$$
\begin{gathered}
\lim _{\left|v_{0}\right| \rightarrow \infty} \mathrm{P}\left(\left|v_{n}\right|^{-1} \leq \tau_{n+1}-\tau_{n} \leq\left|v_{n}\right|^{-1+\delta} \text { for all } n \geq 0\right)=1 \\
\lim _{\left|v_{0}\right| \rightarrow \infty} \mathrm{P}\left(\left(n\left|v_{0}\right|^{-1}+n^{3 / 4}\right)^{1-\delta} \leq \tau_{n} \leq\left(n\left|v_{0}\right|^{-1}+n^{3 / 4}\right)^{1+\delta} \text { for all } n \geq 0\right)=1 .
\end{gathered}
$$


Proof. The first statement easily follows from (29). Then (55) follows from Lemma 6.2 and (54) (considering the cases $\tau_{n}<\left|v_{0}\right|^{3}$ and $\tau_{n} \geq\left|v_{0}\right|^{3}$ separately).

Let $D_{v_{0}}^{\delta}$ be the following event

$$
\begin{gathered}
D_{v_{0}}^{\delta}=\left\{\left(\left|v_{0}\right|+t^{1 / 3}\right)^{1-\delta} \leq|\dot{Y}(t)| \leq\left(\left|v_{0}\right|+t^{1 / 3}\right)^{1+\delta} \quad \text { for all } t \geq 0\right\} \cap \\
\cap\left\{\left|v_{n}\right|^{-1} \leq \tau_{n+1}-\tau_{n} \leq\left|v_{n}\right|^{-1+\delta} \text { for all } n \geq 0\right\} \cap \\
\cap\left\{\left(n\left|v_{0}\right|^{-1}+n^{3 / 4}\right)^{1-\delta} \leq \tau_{n} \leq\left(n\left|v_{0}\right|^{-1}+n^{3 / 4}\right)^{1+\delta} \text { for all } n \geq 0\right\} .
\end{gathered}
$$

As we saw above,

$$
\lim _{\left|v_{0}\right| \rightarrow \infty} \mathrm{P}\left(D_{v_{0}}^{\delta}\right)=1
$$

The next result provides a more precise information about the growth of $\dot{Y}(t)$ but only for a fixed value of $t$.

Lemma 6.4. We have the following limit

$$
\lim _{\left|v_{0}\right| \rightarrow \infty} \liminf _{a \rightarrow \infty} \liminf _{t \rightarrow \infty} \mathrm{P}\left(\frac{1}{a} t^{1 / 3} \leq|\dot{Y}(t)| \leq a t^{1 / 3}\right)=1
$$

Proof. First let us estimate the probability that $|\dot{Y}(t)|$ is too large. To this end, let $0 \leq \delta \leq$ $1 / 4, m$ be the largest integer such that $2^{m} \leq a t^{1 / 3} / 4$, and $n^{*}$ be the first time when $\xi_{n}=m$. Then $\left(t_{n^{*}+1}-t_{n^{*}}\right) / t a^{3}$ is tight by Lemma 5.6. and therefore $\mathrm{P}\left(D_{v_{0}}^{\delta} \cap\left\{t_{n^{*}+1}-t_{n^{*}} \leq t\right\}\right) \rightarrow 0$ as $a \rightarrow \infty$ uniformly in $t \geq 1$. Since $\max _{t \leq t_{n^{*}+1}}|\dot{Y}(t)| \leq a t^{1 / 3}$ on $D_{v_{0}}^{\delta}$ for large $\left|v_{0}\right|$, we see that

$$
\mathrm{P}\left(|\dot{Y}(t)| \geq a t^{1 / 3}\right)
$$

can be made as small as we wish by choosing $a$ and $\left|v_{0}\right|$ large.

Proving that $\dot{Y}(t)$ is unlikely to be small requires a more sophisticated argument. We show that $\dot{Y}$ can not be small on whole $[0, t]$ since this would require that $\dot{Y}$ spends a lot of time near $2^{p}$ for some small $p$ contradicting the transience of $\xi_{n}$. On the other hand Lemma 5.6 allows us to rule out the possibility that $\dot{Y}(t)$ is small while $\dot{Y}(s)$ is large for some $s \in[0, t]$ since 0 is an inaccessible point for $\bar{V}(t)$.

Let us give the precise argument. Let $n_{*}=n_{*}(b, t)$ be the first time when $\left|\dot{Y}\left(t_{n}\right)\right| \geq$ $b t^{1 / 3}$. To estimate the probability that $|\dot{Y}(t)|$ is too small, it is enough to show that

$$
\lim _{\left|v_{0}\right| \rightarrow \infty} \liminf _{b \rightarrow 0} \liminf _{t \rightarrow \infty} \mathrm{P}\left(t_{n_{*}} \leq t\right)=1
$$

since, by Lemma 5.6, for fixed $b$

$$
\mathrm{P}\left(\min _{s \in\left[t_{n_{*}}, t_{n_{*}}+t\right]}|\dot{Y}(s)| \leq \frac{t^{1 / 3}}{a}\right)
$$

can be made as small as we wish by taking $a$ large uniformly in $t \geq 1$. 
Let

$$
T(p)=\sum_{n=1}^{\infty}\left(t_{n+1}-t_{n}\right) \chi_{\left\{\xi_{n}=p\right\}} .
$$

Let $p_{*}=\log _{2}\left(b t^{1 / 3}\right)+2$. Observe that on $D_{v_{0}}^{\delta}$ we have

$$
(1-\delta) \log _{2}\left|v_{0}\right| \leq \xi_{n} \leq p_{*} \text { for } n \leq n_{*} .
$$

Let

$$
F_{v_{0}, K}=\left\{T(p) \leq K 2^{3 p} \times 2^{p_{*}-p} \text { for all } p \text { such that }(1-\delta) \log _{2}\left|v_{0}\right| \leq p \leq p_{*}\right\} .
$$

We claim that for each $N>0$ there are $c_{0}>0, c<1, p_{0}>0$ such that for $p \geq p_{0}$, all $k$ and $v_{0}$ we have

$$
\mathrm{P}\left(T(p) \geq 2^{3 p} k\right) \leq c_{0} \sqrt{k}\left(c^{\sqrt{k}}+2^{-N p}\right) .
$$

Note that (57) implies that for each $\varepsilon>0$ there exist constants $K, r$ such that for $\left|v_{0}\right| \geq r$ we have $P\left(F_{v_{0}, K}\right) \geq 1-\varepsilon$. Also note that (57) implies (56) since on $D_{v_{0}}^{\delta} \cap F_{v_{0}, K}$ we have for all sufficiently large $\left|v_{0}\right|$

$$
t_{n_{*}} \leq \sum_{p=(1-\delta) \log _{2}\left|v_{0}\right|}^{p_{*}} K 2^{3 p} \times 2^{p_{*}-p} .
$$

To establish (57) we note that

$$
\mathrm{P}\left(\#\left(n: \xi_{n}=p\right) \geq \sqrt{k}\right) \leq c^{\sqrt{k}}
$$

since every time $\xi_{n}$ visits $p$ it has a positive probability of never returning there. On the other hand by Lemma 6.1(b)

$$
\mathrm{P}\left(\max _{n: \xi_{n}=p}\left(t_{n+1}-t_{n}\right) \geq 2^{3 p} \sqrt{k} \mid \#\left(n: \xi_{n}=p\right)<\sqrt{k}\right) \leq \sqrt{k}\left(c^{\sqrt{k}}+2^{-N p}\right)
$$

so (57) follows.

\subsection{Probability of a Near Self-Intersection for $Y(t)$}

In this section we prove that if $\left|v_{0}\right|$ is large, then with high probability the 'tail' of the the trajectory $Y(t)$ (the part of the trajectory corresponding to $t \geq \tau_{n}$ ) leaves a neighborhood of $Y_{n}$ and then never comes close to the part of the trajectory corresponding to $t \leq \tau_{n}$. This allows us to conclude that switching to a new version of the force field at each of the times $\tau_{n}$ does not have a major effect on the distribution of the solution, that is the distributions of $X(t)$ and $Y(t)$ are the same if we throw out events of small measure from their respective probability spaces. 
Let $\gamma_{n}, n \geq 1$, be the trajectory of the process $Y(t)$ between times $\tau_{n-1}$ and $\tau_{n}$, that is

$$
\gamma_{n}=\left\{Y(t), \tau_{n-1} \leq t \leq \tau_{n}\right\}
$$

Let $\Gamma_{n}$ be the trajectory of the process after time $\tau_{n}$, that is

$$
\Gamma_{n}=\left\{Y(t), \tau_{n} \leq t<\infty\right\}
$$

Let $\gamma_{n}^{2 R}$ be the $2 R$-neighborhood of $\gamma_{n}$ and $\Gamma_{n}^{R}$ the $R$-neighborhood of $\Gamma_{n}$. We shall prove the following lemma.

Lemma 6.5. There exists $0<\delta<1$ such that

$$
\mathrm{P}\left(D_{v_{0}}^{\delta} \cap \gamma_{n}^{2 R} \cap \Gamma_{n+1}^{R} \neq \emptyset\right) \leq\left(\left|v_{0}\right|+n^{1 / 4}\right)^{-4 d+12-\delta}
$$

for all sufficiently large $\left|v_{0}\right|$ and all $n \geq 1$.

Before we prove Lemma 6.5, let us make several remarks which will, in particular, allow us to deduce parts (a), (b) and (c) of Lemma 4.1 from Lemma 6.5. For $x, v \in \mathbb{R}^{d}$, let $K^{+}(x, v)$ and $K^{-}(x, v)$ be the cones

$$
\begin{gathered}
K^{+}(x, v)=\left\{y \in \mathbb{R}^{d}:(y-x, v) \geq \frac{3}{4}|y-x||v|\right\}, \\
K^{-}(x, v)=\left\{y \in \mathbb{R}^{d}:(y-x,-v) \geq \frac{3}{4}|y-x||v|\right\} .
\end{gathered}
$$

From the definition of $D_{v_{0}}^{\delta}$ it easily follows that

$$
\mathrm{P}\left(D_{v_{0}}^{\delta} \cap \bigcup_{n}\left(\left\{\gamma_{n} \nsubseteq K^{-}\left(Y_{n}, v_{n}\right)\right\} \cup\left\{\gamma_{n+1} \nsubseteq K^{+}\left(Y_{n}, v_{n}\right)\right\}\right)\right) \leq\left|v_{0}\right|^{-N}
$$

if $\left|v_{0}\right|$ is sufficiently large. This implies that for each $0<\delta<1$

$$
\mathrm{P}\left(D_{v_{0}}^{\delta} \cap \bigcup_{n}\left\{\gamma_{n}^{2 R} \cap \gamma_{n+1}^{R} \nsubseteq B_{2 R}\left(Y_{n}\right)\right\}\right) \leq\left|v_{0}\right|^{-N}
$$

for all sufficiently large $\left|v_{0}\right|$. Take $0<\delta<1$ such that (158) holds. Let

$$
\Omega_{v_{0}}=D_{v_{0}}^{\delta} \cap\left\{\gamma_{n}^{2 R} \cap \Gamma_{n+1}^{R}=\emptyset \text { for all } n\right\} \cap\left\{\gamma_{n}^{2 R} \cap \gamma_{n+1}^{R} \subseteq B_{2 R}\left(Y_{n}\right) \text { for all } n\right\} \text {. }
$$

In order to see that parts (a) and (b) of Lemma 4.1 hold, it remains to note that

$$
\lim _{\left|v_{0}\right| \rightarrow \infty} \sum_{n=1}^{\infty}\left(\left|v_{0}\right|+n^{1 / 4}\right)^{-4 d+12-\delta}=0
$$


if $d \geq 4$. From the definition of $D_{v_{0}}^{\delta}$ it immediately follows that $\lim _{t \rightarrow \infty}|\dot{Y}(t)|=\infty$ on $\Omega_{v_{0}}$. Furthermore, the trajectory $Y(t)$ cannot have limit points in $\mathbb{R}^{d}$, as follows from the definition of $\Omega_{v_{0}}$. Therefore, $\lim _{t \rightarrow \infty}|Y(t)|=\infty$, which proves part (c) of Lemma 4.1,

Let us now return to Lemma 6.5. From the definition of $D_{v_{0}}^{\delta}$ it follows that if $\delta^{\prime}>0$, then $\gamma_{n} \subseteq B\left(Y\left(\tau_{n}\right), v_{n}^{\delta^{\prime}}\right)$ for all $n \geq 1$ if $\delta>0$ is sufficiently small and $\left|v_{0}\right|$ is sufficiently large. Let us represent $\Gamma_{n+1}^{R}$ as follows

$$
\Gamma_{n+1}^{R}=\bar{\Gamma}_{n+1}^{R}(\delta) \cup \overline{\bar{\Gamma}}_{n+1}^{R}(\delta)
$$

where $\bar{\Gamma}_{n+1}^{R}(\delta)$ is the $R$-neighborhood of $\bar{\Gamma}_{n+1}(\delta)=\left\{Y(t), \tau_{n+1} \leq t \leq \tau_{n}+\left|v_{n}\right|^{3-\delta}\right\}$ and $\overline{\bar{\Gamma}}_{n+1}^{R}(\delta)$ is the $R$-neighborhood of $\overline{\bar{\Gamma}}_{n+1}(\delta)=\left\{Y(t), \tau_{n}+\left|v_{n}\right|^{3-\delta} \leq t \leq \infty\right\}$.

Recall that the constant $l$ from the definition of the stopping time $\tau_{n}$ is equal to $4 R+1$. Since Lemma 5.10 is obviously also applicable to the process $Z(t)$,

$$
\mathrm{P}\left(D_{v_{0}}^{\delta} \cap\left\{\operatorname{dist}\left(K^{-}\left(Y_{n}, v_{n}\right), \bar{\Gamma}_{n+1}(\delta)\right) \leq 3 R\right\}\right) \leq\left(\left|v_{0}\right|+n^{1 / 4}\right)^{-4 d+12-\delta}
$$

if $\delta>0$ is sufficiently small and $\left|v_{0}\right|$ is sufficiently large. This implies (58) with $\bar{\Gamma}_{n+1}^{R}(\delta)$ instead of $\Gamma_{n+1}^{R}$. Thus, Lemma 6.5 will follow if we prove that

$$
\mathrm{P}\left(D_{v_{0}}^{\delta} \cap \gamma_{n}^{2 R} \cap \overline{\bar{\Gamma}}_{n+1}^{R}(\delta) \neq \emptyset\right) \leq\left(\left|v_{0}\right|+n^{1 / 4}\right)^{-4 d+12-\delta}
$$

Lemma 6.6. There exist $0<\varepsilon<1$ and $0<\delta_{0}<1$ such that for each $0<\delta, \delta^{\prime}<\delta_{0}$ and $R^{\prime}$ the inequality

$$
\mathrm{P}\left(D_{v_{0}}^{\delta} \cap\left\{\left|Y\left(\tau_{n}+\left|v_{n}\right|^{3-\delta}+t\right)-x\right| \leq R^{\prime}\right\} \mid \mathcal{G}_{n}\right) \leq\left(\left|v_{n}\right|^{3-\delta}+t\right)^{-\frac{4}{3}(d-2)-\varepsilon}
$$

holds for all sufficiently large $\left|v_{0}\right|$ uniformly in $n \geq 0, x \in B\left(Y\left(\tau_{n}\right), v_{n}^{\delta^{\prime}}\right)$ and $t \geq 0$.

Proof. In view of Lemma 5.10 we can assume that $t>\left|v_{n}\right|^{3-\delta}$. Denote $\widetilde{t}=\tau_{n}+\left|v_{n}\right|^{3-\delta}+t$. Let us first explain the proof of a weaker bound: for each $\varepsilon>0$ we have

$$
\mathrm{P}\left(D_{v_{0}}^{\delta} \cap\left\{|Y(\widetilde{t})-x| \leq R^{\prime}\right\} \mid \mathcal{G}_{n}\right) \leq t^{-\frac{4}{3}(d-2)+\varepsilon} .
$$

This suffices for $d>4$ (see the proof of Lemma 6.5). Then we explain how to improve this estimate to get (63). The proof of (64) consists of two steps.

(I) Fix $\varepsilon_{1}>0$. We show that if the intersection does take place and $D_{v_{0}}^{\delta}$ takes place then with high probability there exists a number $k$ such that $\tau_{n}+t^{1-\varepsilon_{1}} \leq \tau_{n+k} \leq \widetilde{t}$ and the following conditions are satisfied.

(A) $\left|Y\left(\tau_{n+k}\right)-x\right| \geq t^{4 / 3-\varepsilon}$,

(B) $\frac{\pi}{4} \leq \angle\left(\left(Y\left(\tau_{n+k}\right)-x\right), v_{n+k}\right) \leq \frac{3 \pi}{4}$.

(II) By step (I) it suffices to show that

$$
\mathrm{P}\left(Y(\widetilde{t}) \in B\left(x, R^{\prime}\right) \text { and }(\mathrm{A}) \text { and }(\mathrm{B}) \text { hold }\right) \leq \text { Const }^{-\frac{4}{3}(d-2)+\varepsilon}
$$


To prove (65), denote $r=\left|Y\left(\tau_{n+k}\right)-x\right|$, let $\Pi$ be the plane passing through $x$ orthogonal to $v_{0}$ and let $\operatorname{Pr}$ denote the projection to $\Pi$. We can find a set $S=\left\{x_{j}\right\}$ of cardinality at least $\mathrm{cr}^{d-2}$ such that $x_{1}=x$, the balls $B\left(x_{j}, R^{\prime}\right)$ are disjoint, and for each $j$ there is an isometry $\mathcal{O}_{j}$ leaving $Y\left(\tau_{n+k}\right)$ and $v_{n+k}$ fixed and such that $\mathcal{O}_{j}\left(x_{j}\right)=x_{1}$. By the rotation invariance,

$$
\mathrm{P}\left(\operatorname{Pr}(Y(\widetilde{t})) \in B\left(x_{1}, R^{\prime}\right)\right) \leq \frac{1}{\operatorname{Card}(S)}
$$

proving (65) .

Thus to complete the proof of (64) it remains to justify step I. Observe that on $D_{v_{0}}^{\delta}$ we have

$$
\begin{gathered}
\left|Y\left(\tau_{n}+t^{1-\varepsilon_{1}}\right)-Y\left(\tau_{n}\right)\right| \leq \text { Const } t^{\left(\frac{4}{3}-\varepsilon_{1}\right)(1+\delta)} \\
\left|Y(\widetilde{t})-Y\left(\widetilde{t}-t^{1-\varepsilon_{1}}\right)\right| \leq \text { Const } t^{\left(\frac{4}{3}-\varepsilon_{1}\right)(1+\delta)}
\end{gathered}
$$

On the other hand the inequality $\angle(\dot{Y}(s),(Y(s)-x)) \leq \frac{\pi}{3}$ for all $s \in\left[\tau_{n}+t^{1-\varepsilon_{1}}, \widetilde{t}-t^{1-\varepsilon_{1}}\right]$ would imply

$$
|| Y\left(\widetilde{t}-t^{1-\varepsilon_{1}}\right)-x|-| Y\left(\tau_{n}+t^{1-\varepsilon_{1}}\right)-x|| \geq \text { Const } t^{4 / 3(1-\delta)}
$$

making intersection impossible if $\varepsilon_{1}>3 \delta$. Thus there exists $t_{1} \in\left[\tau_{n}+t^{1-\varepsilon_{1}}, \widetilde{t}-t^{1-\varepsilon_{1}}\right]$ such that $\angle\left(\dot{Y}\left(t_{1}\right),\left(Y\left(t_{1}\right)-x\right)\right)=\frac{\pi}{3}$. Next with high probability the angle changes less than $\frac{\pi}{12}$ on $\left[t_{1}, t_{1}+t^{\left(1-\varepsilon_{1}\right)(3-\delta)}\right]$. Thus the motion on this interval is well approximated by a straight line and consequently there is $t_{2} \in\left[t_{1}, t_{1}+t^{\left(1-\varepsilon_{1}\right)(3-\delta)}\right]$ such that

$$
\left|Y\left(t_{2}\right)-x\right| \geq \text { Const } t^{\left(1-\varepsilon_{1}\right)(3-\delta)} t^{1 / 3-\delta} .
$$

Taking $k$ to be the first number such that $\tau_{n+k}>t_{2}$ establishes our claim.

Now let us now indicate how to prove the lemma in full generality. We need to prove (65) with $-\varepsilon$ instead of $\varepsilon$ in the right-hand side. In the arguments leading to (65) we only used the projection on the plane orthogonal to $v_{n+k}$. Now we consider the projection of the process onto the $v_{n+k}$ direction. During the time interval between $\widetilde{t}-t^{1 / 10}$ and $\widetilde{t}$ the projection of $\dot{Y}(s)$ can be well-approximated by a martingale, and as such by a timechanged Brownian motion. The time-change is almost linear on this small time interval, and thus the projection of $Y(s)$ is approximated well by the integral of the Brownian motion. This allows us to gain an extra factor of $t^{-2 \varepsilon}$. Observe that the derivation of (66) only involved rotation-invariance, and thus (66) remains valid if we replace the probability in the left-hand side by conditional probability with the condition which involves the projection of the process on the direction of $v_{n+k}$.

Proof of Lemma 6.5. Let

$$
s_{k}^{n}(\delta)=\tau_{n-1}+k\left(\left|v_{n}\right|+n^{1 / 4}\right)^{-3 \delta}, k=0, \ldots,\left[\left(\tau_{n}-\tau_{n-1}\right)\left(\left|v_{n}\right|+n^{1 / 4}\right)^{3 \delta}\right] .
$$

As follows from the definition of $D_{v_{0}}^{\delta}$, for each $R^{\prime}$ these points form an $R^{\prime}$-net in $\gamma_{n}$ if $\left|v_{0}\right|$ is sufficiently large. By applying (63) to $x_{k}^{n}(\delta)=Y\left(s_{k}^{n}(\delta)\right)$, we obtain that

$$
\mathrm{P}\left(D_{v_{0}}^{\delta} \cap\left\{\operatorname{dist}\left(Y\left(\tau_{n}+\left|v_{n}\right|^{3-\delta}+t\right), \gamma_{n}\right) \leq R^{\prime}\right\} \mid \mathcal{G}_{n}\right) \leq
$$




$$
\leq\left(\left|v_{n}\right|^{3-\delta}+t\right)^{-\frac{4}{3}(d-2)-\varepsilon}\left(\left|v_{n}\right|+n^{1 / 4}\right)^{3 \delta}
$$

holds for all sufficiently large $\left|v_{0}\right|$ uniformly in $n \geq 0$ and $t \geq 0$. Since $|\dot{Y}(t)| \leq\left(\left|v_{0}\right|+\right.$ $\left.t^{1 / 3}\right)^{1+\delta}$ on $D_{v_{0}}^{\delta}$, and $R^{\prime}$ was arbitrary,

$$
\begin{aligned}
& \mathrm{P}\left(D_{v_{0}}^{\delta} \cap \gamma_{n}^{2 R} \cap \overline{\bar{\Gamma}}_{n+1}^{R}(\delta) \neq \emptyset \mid \mathcal{G}_{n}\right) \leq \mathrm{P}\left(D_{v_{0}}^{\delta} \cap\left\{\operatorname{dist}\left(\overline{\bar{\Gamma}}_{n+1}(\delta), \gamma_{n}\right) \leq 3 R\right\} \mid \mathcal{G}_{n}\right) \leq \\
& \leq \int_{0}^{\infty}\left(\left|v_{n}\right|^{3-\delta}+t\right)^{-\frac{4}{3}(d-2)-\varepsilon}\left(\left|v_{n}\right|+n^{1 / 4}\right)^{3 \delta}\left(\left|v_{0}\right|+\left(\tau_{n}+\left|v_{n}\right|^{3-\delta}+t\right)^{1 / 3}\right)^{1+\delta} d t
\end{aligned}
$$

holds for all sufficiently large $\left|v_{0}\right|$ uniformly in $n \geq 0$. It follows from the definition of $D_{v_{0}}^{\delta}$ that

$$
\left|v_{n}\right| \leq\left(\left|v_{0}\right|+n^{1 / 4}\right)^{1+3 \delta}
$$

on $D_{v_{0}}^{\delta}$ for all sufficiently large $\left|v_{0}\right|$. Recall that

$$
\tau_{n} \leq\left(n\left|v_{0}\right|^{-1}+n^{3 / 4}\right)^{1+\delta}
$$

on $D_{v_{0}}^{\delta}$ for all sufficiently large $\left|v_{0}\right|$. Since $\varepsilon$ is fixed, these estimates imply that right-hand side of (67) can be made smaller than the right-hand side of (62) by taking a sufficiently small $\delta$.

\section{The Convergence in Distribution}

Here we prove Lemma 4.1(d). Recall that $\Omega_{v_{0}}$ is given by (60).

For fixed $v_{0}$, let us prove that the family of processes $\dot{Y}\left(c^{3} t\right) / c$ is tight, when restricted to the event $\Omega_{v_{0}}$. By the Arzela-Askoli Theorem, it is sufficient to show that for each $T, \varepsilon, \eta>0$ there are $c_{0}$ and $\varkappa>0$ such that

$$
\mathrm{P}\left(\Omega_{v_{0}} \cap\left\{\sup _{0 \leq s \leq t \leq T, t-s \leq \varkappa}\left|\dot{Y}\left(c^{3} t\right) / c-\dot{Y}\left(c^{3} s\right) / c\right|>\varepsilon\right\}\right)<\eta
$$

for $c \geq c_{0}$.

Let $T, \varepsilon, \eta>0$ be fixed. Let $n_{*}=n_{*}(\kappa, c)$ be the first time when $\left|\dot{Y}\left(\tau_{n}\right)\right| \geq \kappa c$. Take $\kappa<\varepsilon / 4$. Define $U_{\kappa, c}(t)=\dot{Y}\left(\tau_{n_{*}}+c^{3} t\right) / c$. By Lemma 5.6, there is $\varkappa>0$ such that

$$
\mathrm{P}\left(\Omega_{v_{0}} \cap\left\{\sup _{0 \leq s \leq t \leq T, t-s \leq \varkappa}\left|U_{\kappa, c}(t)-U_{\kappa, c}(s)\right|>\frac{\varepsilon}{2}\right\}\right)<\eta
$$

for large $c$. Now (68) follows easily.

From Lemma 6.4, the definition of $D_{v_{0}}^{\delta}$, and the tightness established above it follows that for each $T, \varepsilon, \eta>0$ there is $\kappa>0$ such that

$$
\mathrm{P}\left(\Omega_{v_{0}} \cap\left\{\sup _{t \in[0, T]}\left|U_{\kappa, c}(t)-\frac{\dot{Y}\left(t c^{3}\right)}{c}\right| \geq \varepsilon\right\}\right)=
$$




$$
\mathrm{P}\left(\Omega_{v_{0}} \cap\left\{\sup _{t \in[0, T]}\left|\frac{\dot{Y}\left(\tau_{n_{*}}+t c^{3}\right)}{c}-\frac{\dot{Y}\left(t c^{3}\right)}{c}\right| \geq \varepsilon\right\}\right)<\eta
$$

for all sufficiently large $c$. Likewise, if $\bar{\tau}_{\kappa}$ is the first time when $|\bar{V}(\bar{\tau})|=\kappa$, define $\bar{U}_{\kappa}(t)=\bar{V}\left(\bar{\tau}_{\kappa}+t\right)$. Then for each $T, \varepsilon, \eta>0$ there is $\kappa>0$ such that

$$
\mathrm{P}\left(\sup _{t \in[0, T]}\left|\bar{U}_{\kappa}(t)-\bar{V}(t)\right| \geq \varepsilon\right)<\eta .
$$

Finally, from Lemma 5.6 and the definition of $\Omega_{v_{0}}$ it follows that the distribution of $U_{\kappa, c}$, considered over the space $\Omega_{v_{0}}$ with the normalized measure, is close to the distribution of $\bar{U}_{\kappa}$ if $c$ is large enough. This completes the proof of Lemma 4.1.

\section{Appendix}

Here we sketch the proof of Lemma 5.3. Note that it is sufficient to prove (33), since (34) follows from (33) in the same way as Corollary 5.2 follows from Lemma 5.1 . We use the same notations as in the proof of Lemma 5.1. It is clear that $\eta_{1} \leq T_{0}=\left|v_{0}\right|^{\alpha}$ with high probability. Therefore, due to (28) and (31) it suffices to show that

$$
\mathrm{E}\left(\left|\int_{\tau_{1}}^{\eta_{1}} \tilde{F}_{0}\left(z\left(s_{0}\right)\right) d s\right| \chi_{\left\{\max \left(\tau_{1}, \eta_{1}\right) \leq T_{0}\right\}}\right) \leq\left|v_{0}\right|^{-3+\delta}
$$

for all sufficiently large $\left|v_{0}\right|$. Since $\tilde{F}$ is a Poisson field, the problem is reduced to showing that for each $\delta>0$ one can choose $\alpha>0$ such that

$$
\mathrm{E}\left(\left|\tau_{1}-\eta_{1}\right| \chi_{\left\{\max \left(\tau_{1}, \eta_{1}\right) \leq T_{0}\right\}}\right) \leq\left|v_{0}\right|^{-3+\delta}
$$

for all sufficiently large $\left|v_{0}\right|$. We shall only prove that

$$
\mathrm{E}\left(\left(\tau_{1}-\eta_{1}\right)^{+} \chi_{\left\{\max \left(\tau_{1}, \eta_{1}\right) \leq T_{0}\right\}}\right) \leq\left|v_{0}\right|^{-3+\delta}
$$

since the inequality with $\eta_{1}-\tau_{1}$ instead of $\tau_{1}-\eta_{1}$ can be proved similarly.

Let $\gamma>0$ and $0 \leq q \leq 2$. We shall specify these constants later. For simplicity of notation, assume that $v_{0}$ is directed along the $x_{1}$-axis, in the positive direction. Let $S_{q, \gamma}$ and $S_{q, \gamma}^{+}$be the following random sets:

$$
\begin{gathered}
S_{q, \gamma}=\left\{x \in \mathbb{R}^{d}: \operatorname{dist}\left(x, z_{0}\left(\eta_{1}\right)\right) \geq 2 R, v_{0} \eta_{1}-2 R \leq x_{1} \leq v_{0} \eta_{1},\right. \\
\left.2 R-\left|v_{0}\right|^{-q+\gamma} \leq \sqrt{x_{2}^{2}+\ldots x_{d}^{2}} \leq 2 R-\left|v_{0}\right|^{-q}+\left|v_{0}\right|^{-2}\right\}, \\
S_{q, \gamma}^{+}=\left\{x \in \mathbb{R}^{d}: \operatorname{dist}\left(x, z_{0}\left(\eta_{1}\right)\right) \geq 2 R, v_{0} \eta_{1}-2 R \leq x_{1} \leq v_{0} \eta_{1},\right. \\
\left.2 R-\left|v_{0}\right|^{-q}+\left|v_{0}\right|^{-2}<\sqrt{x_{2}^{2}+\ldots x_{d}^{2}} \leq 2 R\right\} .
\end{gathered}
$$


Let $\Gamma_{q, \gamma}$ be the following random set:

$$
\Gamma_{q, \gamma}=\left\{x \in \mathbb{R}^{d}: x_{1}=v_{0} \eta_{1}+\left|v_{0}\right|^{-2+2 \gamma+\frac{q}{2}}, \sqrt{x_{2}^{2}+\ldots x_{d}^{2}} \leq\left|v_{0}\right|^{-2+\gamma}\right\} .
$$

Let $U_{q, \gamma}$ be the following random set:

$$
U_{q, \gamma}=\left\{x \in \mathbb{R}^{d}: \operatorname{dist}\left(x, z_{0}\left(\eta_{1}\right)\right) \geq 2 R, \operatorname{dist}\left(x, \Gamma_{q, \gamma}\right) \leq 2 R\right\} .
$$

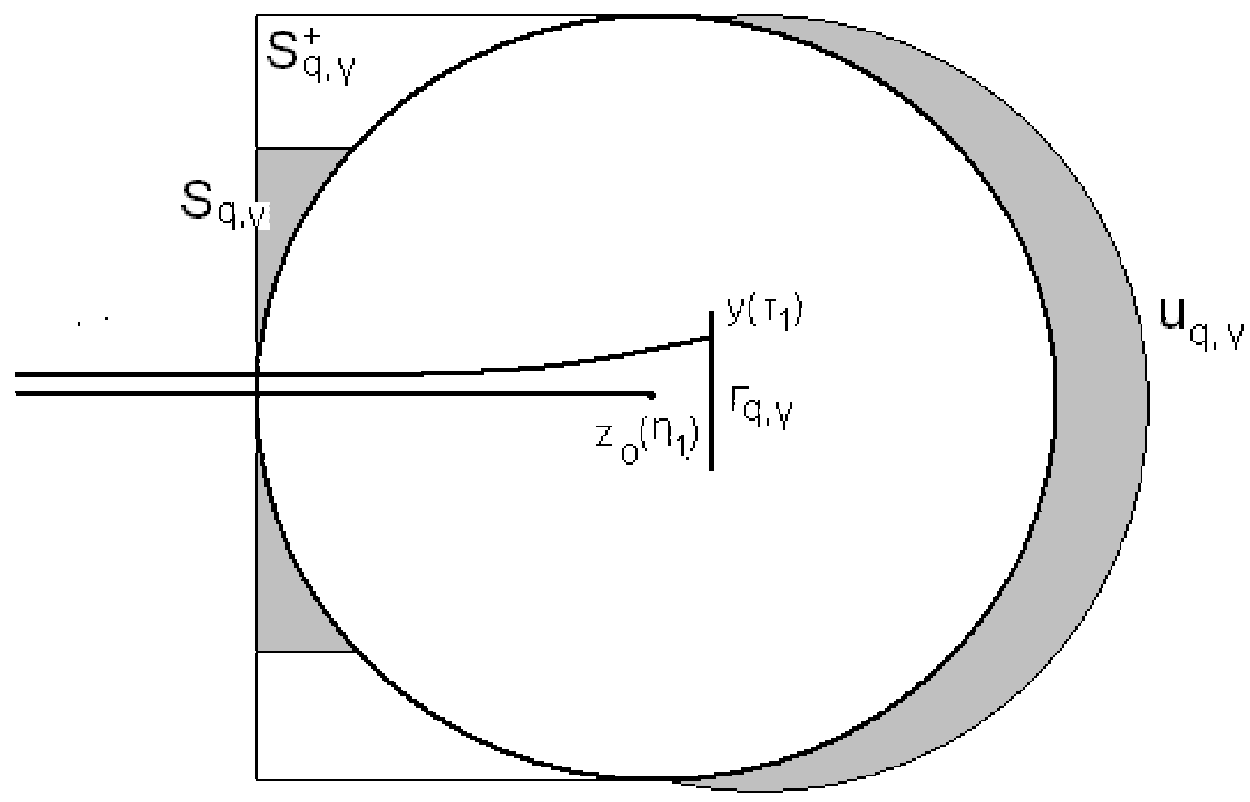

Let $\mathcal{E}_{q, \gamma}^{S}$ be the event that at least one of the points $r_{1}, r_{2}, \ldots$ belongs to $S_{q, \gamma}$ but none belong to $S_{q, \gamma}^{+}$. Let $\mathcal{E}_{q, \gamma}^{U}$ be the event that at least one of the points $r_{1}, r_{2}, \ldots$ belongs to $U_{q, \gamma}$. Let $A$ be a point on the semi-axis $\left\{x \in \mathbb{R}^{d}: x_{1} \geq 0, x_{2}=\ldots=x_{d}=0\right\}$. Note that $\mathcal{E}_{q, \gamma}^{S}$ and $\mathcal{E}_{q, \gamma}^{U}$ are independent when conditioned on $\left\{z_{0}\left(\eta_{1}\right)=A\right\}$. The respective conditional probabilities can be estimated from above by $\left|v_{0}\right|^{-q+2 \gamma}$ and $\left|v_{0}\right|^{-2+3 \gamma+\frac{q}{2}}$ for all sufficiently large $\left|v_{0}\right|$. Therefore, $\mathrm{P}\left(\mathcal{E}_{q, \gamma}^{S} \cap \mathcal{E}_{q, \gamma}^{U}\right) \leq\left|v_{0}\right|^{-2+5 \gamma-\frac{q}{2}}$.

Let us examine the contribution to the expectation (69) from the event $\mathcal{E}_{q, \gamma}^{S}$. First,

$$
\mathrm{E}\left(\chi_{\mathcal{E}_{q, \gamma}^{S} \cap \mathcal{E}_{q, \gamma}^{U}}\left(\tau_{1}-\eta_{1}\right)^{+} \chi_{\left\{\max \left(\tau_{1}, \eta_{1}\right) \leq T_{0}\right\}}\right) \leq T_{0} \mathrm{P}\left(\mathcal{E}_{q, \gamma}^{S} \cap \mathcal{E}_{q, \gamma}^{U}\right) \leq\left|v_{0}\right|^{\alpha-2+5 \gamma-\frac{q}{2}} .
$$

Note that the power $\alpha-2+5 \gamma-\frac{q}{2}$ can be made less than $-3+\delta$ by selecting small $\gamma$ and $\alpha$ close to -1 . Next, note that with high probability the trajectory $y(t)$ reaches the set $\Gamma_{q, \gamma}$ between times $\eta_{1}$ and $\eta_{1}+\left|v_{0}\right|^{-3+3 \gamma+\frac{q}{2}}$ due to the proximity of $y(t)$ and $z_{0}(t)$. Note that the distance between $\Gamma_{q, \gamma}$ and $S_{q, \gamma}^{+}$is greater than $2 R$. Therefore, on $\mathcal{E}_{q, \gamma}^{S} \backslash \mathcal{E}_{q, \gamma}^{U}$, none of the points $r_{1}, r_{2}, \ldots$ belongs to the $2 R$-neighborhood of the point where $y(t)$ first intersects $\Gamma_{q, \gamma}$. Therefore, for each $N>0$,

$$
\mathrm{E}\left(\chi_{\mathcal{E}_{q, \gamma}^{S} \backslash \mathcal{E}_{q, \gamma}^{U}}\left(\tau_{1}-\eta_{1}\right)^{+} \chi_{\left\{\max \left(\tau_{1}, \eta_{1}\right) \leq T_{0}\right\}}\right) \leq\left|v_{0}\right|^{-3+3 \gamma+\frac{q}{2}} \mathrm{P}\left(\mathcal{E}_{q, \gamma}^{S}\right)+\left|v_{0}\right|^{-N} \leq\left|v_{0}\right|^{-3+6 \gamma-\frac{q}{2}} .
$$


Again, the power $-3+6 \gamma-\frac{q}{2}$ can be made less than $-3+\delta$ by selecting small $\gamma$. We have thus obtained that

$$
\mathrm{E}\left(\chi_{\mathcal{E}_{q, \gamma}^{S}}\left(\tau_{1}-\eta_{1}\right)^{+} \chi_{\left\{\max \left(\tau_{1}, \eta_{1}\right) \leq T_{0}\right\}}\right) \leq\left|v_{0}\right|^{-3+\delta} .
$$

Note that for fixed $\gamma$ one can find finitely many numbers $q_{1}, \ldots, q_{n} \in[0,2]$ such that $\mathrm{P}\left(\bigcup_{i=1}^{n} \mathcal{E}_{q_{i}, \gamma}^{S}\right)=1$. Therefore, (70) implies (69) $)$.

\section{References}

[1] Billingsley P. Probability and Measure Wiley-Interscience, 1995.

[2] Dolgopyat D., Fermi Acceleration, Cont. Math 469 (2008) 149-166.

[3] Dolgopyat D., Koralov L. Avergaging of Hamiltonian flows with an ergodic component, to appear in Ann. Prob.

[4] Durr D., Goldstein S., Lebowitz J., Asymptotic Motion of a Classical Particle in a Random Potential in Two Dimensions: Landau Model, Comm. Math. Phys. 113 (1987) 209-230.

[5] Kesten H., Papanicolaou G. A Limit Theorem for Stochastic Acceleration, Comm. Math. Phys. 78 (1980/81) 19-63.

[6] Komorowski T., Ryzhik L. Diffusion in a Weakly Random Hamiltonian Flow, Comm. Math. Phys. 263 (2006) 277-323.

[7] Komorowski T., Ryzhik L. The Stochastic Acceleration Problem in Two Dimensions, Israel Journal of Mathematics, 155 (2006) 157-204.

[8] Karatzas I., Shreve S. Brownian Motion and Stochastic Calculus, 2nd ed., Springer 1994.

[9] Revuz D. and Yor M. Continuous Martingales and Brownian Motion, 3d ed., Sringer, Berlin-Heildelberg-New York, 1998. 